\title{
CONTROLLED BOUNDARY AND $h$-COBORDISM THEOREMS
}

BY

\author{
T. A. CHAPMAN 1
}

\begin{abstract}
In this paper two theorems are established which are consequences of some earlier approximation results of the author. The first theorem is a controlled boundary theorem for finite-dimensional manifolds. By this we mean an ordinary boundary theorem plus small $\varepsilon$-control in a given parameter space. The second theorem is a controlled $h$-cobordism theorem for finite-dimensional manifolds with small $\varepsilon$-control in a given parameter space. These results generalize the End Theorem and the Thin $h$-Corbordism of Quinn.
\end{abstract}

1. Introduction. The purpose of this paper is to establish two theorems which are consequences of the approximation results of [2]. The first theorem is a controlled boundary theorem for finite-dimensional manifolds. By this we mean an ordinary boundary theorem, as treated in Siebenmann [8], plus small $\varepsilon$-control in a given parameter space. The second theorem is a controlled $h$-cobordism theorem for finite-dimensional manifolds with similar small $\varepsilon$-control in a given parameter space. These results generalize the End Theorem and the Thin $h$-Cobordism Theorem of Quinn [7] in the sense that they do not require the parameter space to be locally simply connected and they do not require that the map to the parameter space be locally constant on $\pi_{1}$. This is accomplished by replacing the stable algebra (i.e. geometric module) approach of Quinn $[7, \S 8]$ by the more topological approximation results of [2].

Thoughout this paper $B$ will always be a finite-dimensional compact metric space. If $M^{m}$ is a noncompact (TOP or PL) manifold and $p: M \rightarrow B$ is a map, then we say that $M$ admits a controlled boundary if $p$ continuously extends to $\tilde{p}: \tilde{M} \rightarrow B$, where $\tilde{M}$ is a compact manifold and $M=\tilde{M}-\partial \tilde{M}$. Our Controlled Boundary Theorem below imposes three conditions on $M$ in order for it to admit such a controlled boundary. The first two are necessary, while the third (a local $\pi_{1}$ requirement) is not. We first describe the necessary conditions.

We say that $p: M \rightarrow B$ is tame at $\infty$ provided that given any $\varepsilon>0$ and compactum $C \subset M$ there exists a larger compactum $D \supset C$ and a homotopy $h_{t}: M-C$ $\rightarrow M-C$ such that $h_{0}=\mathrm{id}, h_{1}(M-C) \subset D-C$, and $p h_{t}: M-C \rightarrow B$ is an $\varepsilon$ homotopy. This latter requirement means that the diameter of the track of each point under this homotopy is less than $\varepsilon$. It is obvious that if $M$ admits a controlled boundary, then $p$ must be tame at $\infty$. The second necessary condition is motivated by the condition " $\pi_{1}$ stable at $\infty$ " that was used in Siebenmann [8] to describe the

Received by the editors March 4, 1982.

1980 Mathematics Subject Classification. Primary 57A15, 57A30.

Key words and phrases. Boundary theorem, $h$-cobordism theorem.

${ }^{1}$ Supported in part by an NSF Grant. 
uncontrolled problem. Loosely phrased it means that fundamental groups of complements of large compacta in $M$ can be moved toward $\infty$ with small $\varepsilon$-control in $B$. Specifically we define $p: M \rightarrow B$ to be 1-movable at $\infty$ provided that given any $\varepsilon>0$ and compactum $C \subset M$ there exists a larger compactum $D \supset C$ so that the following property is satisfied: for every compactum $E \supset D$ there exists a larger compactum $F \supset E$ such that if $(K, L)$ is any 2-dimensional compact polyhedral pair and $f:(K, L) \rightarrow(M-D, M-F)$ is a map, then there exists a map $g:(K, L) \rightarrow(M-$ $E, M-F)$ such that:

(1) $g=f$ on $L$,

(2) $p g$ is $\varepsilon$-close to $p f$,

(3) if $K^{1}$ is the 1-skeleton of $K$, then there is a homotopy $h_{t}: f\left|K^{1} \cup L \simeq g\right| K^{1}$ $\cup L$ rel $L$ in $X-C$ for which $p h_{t}$ is an $\varepsilon$-homotopy.

Observe that if we ignore the control in $B$ and assume that everything is connected, then the above properties imply that

$$
\operatorname{image}\left(\pi_{1}(M-E) \rightarrow \pi_{1}(M-C)\right)=\operatorname{image}\left(\pi_{1}(M-D) \rightarrow \pi_{1}(M-C)\right)
$$

and

$$
\operatorname{kernel}\left(\pi_{1}(M-F) \rightarrow \pi_{1}(M-D)\right)=\operatorname{kernel}\left(\pi_{1}(M-F) \rightarrow \pi_{1}(M-E)\right) .
$$

Again it is obvious that if $M$ admits a controlled boundary, then $p$ is 1-movable at $\infty$.

In the sequel Wh will denote the Whitehead group functor, which is a homotopy functor from the category of spaces and homotopy classes of maps to the category of abelian groups and homomorphisms [5, §10]. By direct summing over components this makes good sense even if the spaces are not path connected. We say that $p: M \rightarrow B$ is nice at $\infty$ provided that for every $b \in B$, neighborhood $U \subset B$ of $b$, and compactum $C \subset M$, there exists a smaller neighborhood $V \subset U$ of $b$ and a larger compactum $D \supset C$ for which the inclusion

$$
\left[p^{-1}(V) \cap(M-D)\right] \times T^{k} \hookrightarrow\left[p^{-1}(U) \cap(M-C)\right] \times T^{k}
$$

induces the 0 -homomorphism on Whitehead groups, for all $k$-tori $T^{k}$. (We also allow $p^{-1}(V)$ and $p^{-1}(U)$ to be empty.) A simple example of this is provided by a map $p: M \rightarrow B$ which is $U V^{1}$ at $\infty$, i.e., for any $b \in B$, neighborhood $U \subset B$ of $b$, and compactum $C \subset M$, there exists a neighborhood $V \subset U$ of $b$ and a compactum $D \supset C$ for which any loop in $p^{-1}(V) \cap(M-D)$ is null-homotopic in $p^{-1}(U) \cap(M$ $-C$ ). Such a map is easily seen to be nice at $\infty$ by using the dependence of the Whitehead group functor on $\pi_{1}$, the homotopy functorality of Wh, and the difficult calculation $\mathrm{Wh}\left(T^{k}\right)=0$ [1]. Of course, niceness at $\infty$ is not a necessary condition for $M$ to admit a controlled boundary. It is just a condition imposed on $M$ which is used to conclude that certain obstructions to putting a controlled boundary on $M$ vanish.

Controlled Boundary Theorem. Let $M^{m}$ be a manifold, $m \geqslant 6$ and $\partial M=\varnothing$, and let $p: M \rightarrow B$ be a map which is tame at $\infty, 1-$ movable at $\infty$, and nice at $\infty$. Then $M$ admits a controlled boundary. 
REMARKS. There are also the expected relative versions of this result which are similar to those stated in the Relative End Theorem of [7]. For example if $\partial M \neq \varnothing$ and $\partial M$ already has a controlled boundary, then $M$ can be given a controlled boundary which agrees with the given one on $\partial M$. Also if $C \subset B$ is compact and the portion of $M$ over some neighborhood of $C$ already has a controlled boundary, then $M$ can be given a controlled boundary which agrees with the given one over $C$.

In order to state our Controlled $h$-Cobordism Theorem we will need more definitions. If $W^{m+1}$ is a compact (TOP or PL) manifold and $p: W \rightarrow B$ is a map, then a cobordism $\left(W, \partial_{0} W\right)$ is said to be an $(\varepsilon, h)$-cobordism provided that there are deformation retractions of $W$ to $\partial_{0} W$ and $\overline{\partial W-\partial_{0} W}$ so that the images of these deformations in $B$ are $\varepsilon$-homotopies. Similarly we say that $\left(W, \partial_{0} W\right)$ is an $\varepsilon$-product if there exists a homeomorphism $h:[0,1] \times \partial_{0} W \rightarrow W$ for which $h(0, x)=x$ and $p h$ is $\varepsilon$-close to the composition

$$
p(\text { proj }):[0,1] \times \partial_{0} W \stackrel{\text { proj }}{\rightarrow} \partial_{0} W \stackrel{p}{\rightarrow} B .
$$

For a given $\varepsilon>0$ our Controlled $h$-Cobordism Theorem below imposes conditions which guarantee that there is a small $\delta>0$ for which a $(\delta, h)$-cobordism becomes an $\varepsilon$-product. Here is the basic definition. For any $b \in B$ let $S_{\varepsilon}(b)$ be the open $\varepsilon$-ball around $b$. Then we say that $p: W \rightarrow B$ is $(\varepsilon, \delta)$-nice if given any $b \in B$ the inclusion-induced homomorphism,

$$
\mathrm{Wh}\left(p^{-1}\left(S_{\delta}(b)\right) \times T^{k}\right) \rightarrow \mathrm{Wh}\left(p^{-1}\left(S_{\varepsilon}(b)\right) \times T^{k}\right),
$$

is the 0-homomorphism, for all $k$. A simple example of this is provided by $p=\operatorname{proj}: B \times F \rightarrow B$, where $B$ and $F$ are compact ANR's for which $\pi_{1}(C)$ is free abelian for all path components $C$ of $F$. From the $\pi_{1}$-condition we conclude that $\mathrm{Wh}\left(F \times T^{k}\right)=0$, and from the local contractibility of $B$ (and the homotopy functorality of Wh) we see that for every $\varepsilon>0$ there exists a $\delta>0$ for which $p$ is $(\varepsilon, \delta)$-nice.

Controlled $h$-Cobordism TheOREM. Suppose we are given a compactum $B$, an integer $m \geqslant 5$, and $\varepsilon>0$. There exists a decreasing set $\left\{\delta_{i}\right\}_{i=1}^{k}$ of positive numbers so that if $W^{m+1}$ is a manifold, $p: W \rightarrow B$ is a map which is $\left(\delta_{i}, \delta_{i+1}\right)$-nice for all $i$, and $\left(W, \partial_{0} W\right)$ is a $\left(\delta_{k}, h\right)$-cobordism, then $\left(W, \partial_{0} W\right)$ is an $\varepsilon$-product.

The scope of this result is greatly enhanced by the following addendum which clarifies the manner in which $k$ and the $\delta_{i}$ are chosen.

ADDENDUM. 1. The integer $k$ depends only on $\operatorname{dim} B$.

2. The set $\left\{\delta_{i}\right\}$ clearly depends on $B, m$, and $\varepsilon$, and it can be rechosen as follows: For a fixed $i_{0}$ and any $x \in\left(0, \delta_{i_{0}}\right)$ we can find another set $\left\{\delta_{i}^{\prime}\right\}_{i=1}^{k}$, fulfilling the above requirements, which is of the form $\delta_{i}^{\prime}=\delta_{i}$, for $i<i_{0}$, and $\delta_{i_{0}}^{\prime}=x$.

REMARK. As in the case of the Controlled Boundary Theorem there is also the expected relative version of this result when $\left(W, \partial_{0} W\right)$ has a given controlled product structure over a portion of $B$.

Concerning the proofs of the Controlled Boundary Theorem and the Controlled $h$-Cobordism Theorem we will only give the details for the PL case. As is usually 
done, the TOP case is dealt with by merely reminding the reader that, in the dimension ranges we are concerned with, the appropriate handlebody techniques exist to translate our PL arguments into the TOP category [6]. So in the sequel we will be working only in the PL category.

Finally we make a few remarks concerning the organization of the material in this paper. In $\$ 3$ we establish a controlled Hurewicz theorem which is needed throughout the paper. This is more general than a similar result of [7], for it is designed to specifically apply to our situation in which there is no local control on $\pi_{1}$. 4 contains some notation and observations concerning controlled matrices. It is merely a formulation of the algebraic language needed in $\$ 5$, which deals with a controlled version of Wall's finiteness obstruction theory. This is complicated by the fact that multiple basepoints must be dealt with simultaneously. This is all put together in $\$ 6$ for a proof of the Controlled Boundary Theorem. In $\$ 7$ we prove the Controlled $h$-Cobordism Theorem by using the method of proof of the Controlled Boundary Theorem to split up a given controlled $h$-cobordism into a number of small $h$-cobordisms. In order to pull this off we have to invoke a relative version of the Approximation Theorem of [2].

2. Definitions and notation. The purpose of this section is to introduce some language which will be used throughout this paper. First a word about the use of the term clean. If $X$ is a polyhedron, then a closed subset $A$ of $X$ is said to be clean in $X$ provided that $A$ is a subpolyhedron and the topological boundary of $A, \operatorname{Bd}(A)$, is a subpolyhedron which is $\mathrm{PL}$ bicollared in $X$. This means that $\operatorname{Bd}(A)$ has a $\mathrm{PL}$ collaring in both $A$ and the closure of $X-A, \overline{X-A}$. If $M^{m}$ is a manifold, possibly with boundary, then a closed subset $N^{n}$ of $M$ is clean provided that $N$ is a PL submanifold and $\operatorname{Bd}(N)$ is a PL submanifold which is PL bicollared. This necessarily means that $N \cap \partial M$ is also clean in $\partial M$.

Next we give some definitions which generalize to the controlled setting some standard notions from the homotopy category. In what follows $p: Y \rightarrow B$ will be a controlling map from a space $Y$ to our parameter space $B$. For convenience we say that a homotopy into $Y$ is a $p^{-1}(\varepsilon)$-homotopy provided that the composition of the homotopy with $p$ is an $\varepsilon$-homotopy into $B$. Our first definition is a controlled version of the notion of a homotopy equivalence. We say that a map $f: X \rightarrow Y$ is an $\varepsilon$-equivalence provided that there is a map $g: Y \rightarrow X$ for which $f g$ is $p^{-1}(\varepsilon)$-homotopic to $\mathrm{id}_{Y}$ and $g f$ is $(p f)^{-1}(\varepsilon)$-homotopic to $\mathrm{id}_{X}$. Now generalizing the notion of an $n$-equivalence we say that $f: X \rightarrow Y$ is an $(\varepsilon, n)$-equivalence if for every $k \leqslant n+1$ and commutative diagram

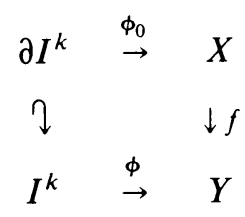

there exists a map $\tilde{\phi}: I^{k} \rightarrow X$ such that $\tilde{\phi} \mid \partial I^{k}=\phi_{0}, d(p f \tilde{\phi}, p \phi)<\varepsilon$, and for each $k \leqslant n$ there exists a $p^{-1}(\varepsilon)$-homotopy $\tilde{f} \tilde{\phi} \simeq \phi$ rel $\partial I^{k}$. Finally generalizing the notion of an $n$-connected map we say that $f: X \rightarrow Y$ is $(\varepsilon, n)$-connected if for every $k \leqslant n$ 
and commutative square as above, there exists a map $\tilde{\phi}: I^{k} \rightarrow X$ such that $\tilde{\phi} \mid \partial I^{k}=\phi_{0}$ and there exists a $p^{-1}(\varepsilon)$-homotopy $\tilde{\phi} \tilde{\phi} \simeq \phi$ rel $\partial I^{k}$.

We will also have to have a notion of spaces which are homotopically dominated by compact polyhedra with $\varepsilon$-control in $B$. If $p: Y \rightarrow B$ is a controlling map as above, then we define $Y$ to be $\varepsilon$-finitely dominated provided that there exist a compact polyhedron $K$ and maps $f: K \rightarrow Y, g: Y \rightarrow K$ such that $f g$ is $p^{-1}(\varepsilon)$-homotopic to $\operatorname{id}_{Y}$. The map $f$ is called an $\varepsilon$-domination and the map $g$ is called a (right) $\varepsilon$-inverse of $f$.

3. A controlled Hurewicz Theorem. Throughout this section $(X, Y)$ will be a pair of locally compact separable metric ANR's and $p: X \rightarrow B$ will be a controlling map to our parameter space. We also assume that $Y$ is compact and $X$ is $\varepsilon$-finitely dominated, for all $\varepsilon>0 .^{2}$ Given that $Y \hookrightarrow X$ is a $(\delta, 1)$-equivalence, for $\delta$ small, the main result of this section establishes a local homology condition which implies that the inclusion $Y \hookrightarrow X$ is $(\varepsilon, n)$-connected, where $\varepsilon$ is a function of $\delta$ and $n$ which can be chosen small if $n$ is held fixed and $\delta$ is small. While a similar result was established in Quinn [7], it appears that our homology condition has a more local character.

Before stating our main result we will have to introduce some more notation. For any ANR $X$ let $X \stackrel{q}{\sim} \underset{\rightarrow}{\rightarrow}$ be the covering space for which $q^{-1}(C) \stackrel{q}{\rightarrow} C$ is the universal covering space of $C$, for each component $C$ of $X$. Whenever convenient we will alternately denote $q^{-1}(A)$ by $A^{-}$and $\tilde{X} \mid A$, for each $A$ in $X$. Now choose a collection of basepoints $\{c\} \subset X$, one for each component of $X$, and define $\pi_{1}(X,\{c\})$ to be the product group, $\Pi\left\{\pi_{1}(X, c) \mid c \in\{c\}\right\}$. For each $c \in\{c\}$ choose $e_{c} \in q^{-1}(c)$. Then the choice of $\left\{e_{c}\right\}$ determines an isomorphism, $\pi_{1}(X,\{c\}) \approx G_{X}$, where $G_{X}$ is the group of covering transformations of $\tilde{X^{\tau}}$. The singular homology group $H_{n}(\tilde{X})$ is naturally a $Z G_{X}$ module, and it is thus a $Z \pi_{1}(X,\{c\})$-module once $\left\{e_{c}\right\}$ has been chosen. For any $A \subset X$ note that $H_{n}(\tilde{X} \mid A)$ and $H_{n}(\tilde{X}, \tilde{X} \mid A)$ are also $Z G_{X}$ modules.

Here is the condition needed in our main result below. We say that the pair $(X, Y)$ is homologically $(\varepsilon, \delta, n)$-trivial, for $\delta \leqslant \varepsilon$, if for every $b \in B$ the inclusion-induced homomorphism

$$
H_{n}\left(p^{-1}\left(S_{\delta}(b)\right)^{\sim},\left(p^{-1}\left(S_{\delta}(b)\right) \cap Y\right)^{\sim}\right) \rightarrow H_{n}\left(p^{-1}\left(S_{\varepsilon}(b)\right)^{\sim},\left(p^{-1}\left(S_{\varepsilon}(b)\right) \cap Y\right)^{\sim}\right)
$$

is 0 . To explain what this homomorphism means we first choose basepoints $\{c\}$ in the components of $p^{-1}\left(S_{\delta}(b)\right)$ and $\left\{e_{c}\right\}$ over $\{c\}$ so that the left-hand side of the above map is a $Z \pi_{1}\left(p^{-1}\left(S_{\delta}(b)\right),\{c\}\right)$-module. Similarly choose basepoints $\{d\} \subset$ $p^{-1}\left(S_{\varepsilon}(b)\right)$ so that $\{c\} \subset\{d\}$ and choose $\left\{f_{d}\right\}$ over $\{d\}$. Then the inclusion

$$
i:\left(p^{-1}\left(S_{\delta}(b)\right), p^{-1}\left(S_{\delta}(b)\right) \cap Y\right) \rightarrow\left(p^{-1}\left(S_{\varepsilon}(b)\right), p^{-1}\left(S_{\varepsilon}(b)\right) \cap Y\right)
$$

is uniquely covered by a map

$$
i^{\sim}:\left(p^{-1}\left(S_{\delta}(b)\right)^{\sim},\left(p^{-1}\left(S_{\delta}(b)\right) \cap Y\right)^{\sim}\right) \rightarrow\left(p^{-1}\left(S_{\varepsilon}(b)\right)^{\sim},\left(p^{-1}\left(S_{\varepsilon}(b)\right) \cap Y\right)^{\sim}\right)
$$

for which $e_{c}$ is taken to $f_{c}$, for all $c \in\{c\}$. This map determines a homomorphism on homology, which is our desired homomorphism. It is important to observe that if

${ }^{2}$ This is the situation encountered in $\$ 6$. 
this homomorphism is 0 for any one choice of $\{c\},\left\{e_{c}\right\},\{d\}$ and $\left\{f_{d}\right\}$, then it is 0 for any other. Here is our main result.

THEOREM 3.1. Let an integer $n \geqslant 2$ and a compact metric space $B$ be given. For every $\varepsilon>0$ there exists a sequence $\left\{\delta_{i}\right\}_{i=1}^{2 n}$ of positive numbers such that if $(X, Y)$ and $p: X \rightarrow B$ are chosen so that $(X, Y)$ is homologically $\left(\delta_{2 i}, \delta_{2 i-1}, i\right)$-trivial, for $1 \leqslant i \leqslant$ $n$, and $Y \hookrightarrow X$ is a $\left(\delta_{1}, 1\right)$-equivalence, then $(X, Y)$ is $(\varepsilon, n)$-connected.

As in the case of the Controlled $h$-Cobordism Theorem there is an addendum which further explains how the $\delta_{i}$ are chosen.

ADDENDUM. The set $\left\{\delta_{i}\right\}$ can be rechosen as follows: For a fixed $i_{0}$ and any $x \in\left(0, \delta_{i_{0}}\right)$ we can find another set $\left\{\delta_{i}^{\prime}\right\}_{i=1}^{2 n}$, fulfilling the above requirements, which is of the form $\delta_{i}^{\prime}=\delta_{i}$, for $i>i_{0}$, and $\delta_{i_{0}}^{\prime}=x$.

We now begin the proof of Theorem 3.1. We will assume that $(X, Y)$ is a compact relative $\mathrm{CW}$ pair, i.e., $X$ is obtained from $Y$ by attaching a finite number of cells. After this special case has been taken care of we will show how the argument can be modified to obtain the more general case. The following result gives the basic geometric connection between homology and homotopy that will be needed in our argument. For a proof see $[5, \S 8]$.

LEMma 3.2. Assume that $X$ is obtained from $Y$ by attaching cells of dimensions $\geqslant n$ $(n \geqslant 2)$, where 2-cells are trivially attached. If $\{c\} \subset Y$ is a collection of basepoints and $\left\{e_{c}\right\} \subset \bar{X}$ are chosen over $\{c\}$, then there is a $Z \pi_{1}(X,\{c\})$-module isomorphism

$$
\pi_{n}(X, Y,\{c\})=\sum\left\{\pi_{n}(X, Y, c) \mid c \in\{c\}\right\} \approx H_{n}(\tilde{X}, \tilde{Y})
$$

which is uniquely determined by the choice of $\left\{e_{c}\right\}$.

COROllary 3.3. Given the above data assume that each cell of $X$ lies in some $p^{-1}\left(S_{\delta}(b)\right)$ and assume that $(X, Y)$ is homologically $(\varepsilon, \delta, n)$-trivial. Then for each $n$-cell of $X$ in $p^{-1}\left(S_{\delta}(b)\right)$ there is a homotopy of this $n$-cell rel $\partial$ into $Y$, with this homotopy taking place in $p^{-1}\left(S_{\varepsilon+2 \delta}(b)\right)$.

Proof. Let $\phi:\left(I^{n}, \partial I^{n}\right) \rightarrow\left(p^{-1}\left(S_{\delta}(b)\right), p^{-1}\left(S_{\delta}(b)\right) \cap Y\right)$ be a characteristic map for the given $n$-cell of $X$. Define $Z_{0}$ to be $p^{-1}\left(S_{\varepsilon+2 \delta}(b)\right) \cap Y$ and let $Z$ be the union of $Z_{0}$ and all cells of $X$ which meet $p^{-1}\left(S_{\varepsilon}(b)\right)$. It then follows that $Z \cap Y=Z_{0}$. We may regard the homotopy class $[\phi]$ as an element of $\pi_{n}\left(p^{-1}\left(S_{\delta}(b)\right), p^{-1}\left(S_{\delta}(b)\right) \cap Y\right)$, and all we have to do is show that its inclusion-induced image in $\pi_{n}\left(Z, Z_{0}\right)$ is 0 . (For simplicity we have suppressed any mention of basepoints in the various components.) By the idea of Lemma 3.2 above we have an isomorphism $\pi_{n}\left(Z, Z_{0}\right) \approx H_{n}\left(\tilde{Z}, \tilde{Z_{0}}\right)$, and by the same argument we have a homomorphism of

$$
\pi_{n}\left(p^{-1}\left(S_{\delta}(b)\right), p^{-1}\left(S_{\delta}(b)\right) \cap Y\right)
$$

to $H_{n}\left(p^{-1}\left(S_{\delta}(b)\right) \tilde{},\left(p^{-1}\left(S_{\delta}(b)\right) \cap Y\right)^{\tilde{}}\right)$ for which the following diagram commutes:

$$
\begin{array}{ccc}
\pi_{n}\left(p^{-1}\left(S_{\delta}(b)\right), p^{-1}\left(S_{\delta}(b)\right) \cap Y\right) & \rightarrow & \pi_{n}\left(Z, Z_{0}\right) \\
\downarrow & & \downarrow \approx \\
H_{n}\left(p^{-1}\left(S_{\delta}(b)\right)^{\sim},\left(p^{-1}\left(S_{\delta}(b)\right) \cap Y\right)^{\sim}\right) & \rightarrow & H_{n}\left(Z^{\sim}, Z_{0}^{\sim}\right)
\end{array}
$$


The bottom arrow is inclusion-induced, and it must be 0 since $(X, Y)$ is homologically $(\varepsilon, \delta, n)$-trivial. Thus the image of $[\phi]$ in $\pi_{n}\left(Z, Z_{0}\right)$ is 0 .

Our proof of Theorem 3.1 uses some observations on cell-trading which are explained in the statements below. For notation let $\phi:\left(I^{n}, \partial I^{n}\right) \rightarrow(X, Y)$ be a characteristic map for a cell of $X$ for which there exists a homotopy $\phi \simeq \psi \operatorname{rel} \partial I^{n}$ such that $\psi\left(I^{n}\right) \subset Y$. As explained in [5, §7] the homotopy $\phi \simeq \psi$ can be used to trade this $n$-cell for a $(n+2)$-cell, thereby obtaining a compact relative $\mathrm{CW}$ pair $\left(X_{1}, Y\right)$ and a homotopy equivalence $g: X \rightarrow X_{1}$ rel $Y$ which preserves skeleta. The following lemma is merely a result of keeping track of how much stretching is involved when the homotopy $\phi \simeq \psi$ has specified control in $B$.

LEMMA 3.4. For every $\gamma>0$ there exists a $\mu>0$ so that if the homotopy $\phi \simeq \psi$ is a $p^{-1}(\mu)$-homotopy, then there is a $\gamma$-equivalence $f: X_{1} \rightarrow X \operatorname{rel} Y$ with $\gamma$-inverse $g$ as mentioned above. Moreover there is an integer $k$ (depending on none of the above data) for which $\gamma=k \mu$.

Corollary 3.5. Given the $\gamma$-equivalence above let $p_{1}=p f: X_{1} \rightarrow B$ and let $g: X \rightarrow$ $X_{1}$ be the given $\gamma$-inverse of $f$. It follows that

(1) if $(X, Y)$ is homologically $(\varepsilon, \delta, n)$-trivial, then $\left(X_{1}, Y\right)$ is homologically $(\varepsilon+$ $2 \gamma, \delta, n)$-trivial,

(2) if $A \subset X$ and diam $p(A)<\varepsilon$, then diam $p_{1} g(A)<\varepsilon+2 \gamma$.

Proof. (1) For any $b \in B$ observe that the inclusion $p_{1}^{-1}\left(S_{\delta}(b)\right) \hookrightarrow p_{1}^{-1}\left(S_{\varepsilon+2 \gamma}(b)\right)$ factors (up to homotopy) into

$$
p_{1}^{-1}\left(S_{\delta}(b)\right) \stackrel{f}{\rightarrow} p^{-1}\left(S_{\delta}(b)\right) \hookrightarrow p^{-1}\left(S_{\varepsilon}(b)\right) \stackrel{g}{\rightarrow} p_{1}^{-1}\left(S_{\varepsilon+2 \gamma}(b)\right) .
$$

(2) For any $x_{1}, x_{2} \in X$ observe that

$$
\begin{aligned}
& d\left(p_{1} g\left(x_{1}\right), p_{1} g\left(x_{2}\right)\right) \\
& \quad \leqslant d\left(p_{1} g\left(x_{1}\right), p\left(x_{1}\right)\right)+d\left(p\left(x_{1}\right), p\left(x_{2}\right)\right)+d\left(p\left(x_{2}\right), p_{1} g\left(x_{2}\right)\right) .
\end{aligned}
$$

Proof of Theorem 3.1. It will suffice to consider the case $n=2$. First assume that the cells of $X$ have been subdivided sufficiently so that they are small, i.e., their images in $B$ have small diameters. We will assume that numbers $\delta_{1}<\delta_{2}<\delta_{3}<\delta_{4}$ are given so that $(X, Y)$ is homologically $\left(\delta_{4}, \delta_{3}, 2\right)$-trivial and $Y \hookrightarrow X$ is a $\left(\delta_{1}, 1\right)$ equivalence. (The condition that $(X, Y)$ is homologically $\left(\delta_{2}, \delta_{1}, 1\right)$-trivial is redundant.) We will show that if these numbers are appropriately chosen, then $(X, Y)$ must be $(\varepsilon, 2)$-connected.

Using the fact that $Y \hookrightarrow X$ is a $\left(\delta_{1}, 1\right)$-equivalence we can trade the 0 -cells for 2-cells and the 1-cells for 3-cells. By Lemma 3.4 this gives us a relative $\mathrm{CW}$ complex $\left(X_{1}, Y\right)$ and a $\gamma_{1}$-equivalence $f_{1}: X_{1} \rightarrow X$, where $\gamma_{1}$ is small provided that $\delta_{1}$ is correspondingly small. Now $p_{1}=p f: X_{1} \rightarrow B$ is our controlling map and it follows from Corollary 3.5 that the cells of $X_{1}$ are small (say diameter $\ll \delta_{3}$ in $B$ ) and $\left(X_{1}, Y\right)$ is homologically $\left(\delta_{4}+2 \gamma_{1}, \delta_{3}, 2\right)$-trivial. Since $f_{1}: X_{1} \rightarrow X$ is a $\gamma_{1}$-equivalence and $Y \hookrightarrow X$ is a $\left(\delta_{1}, 1\right)$-equivalence, it follows that $Y \hookrightarrow X_{1}$ is a $\left(\delta_{1}+\gamma_{1}, 1\right)$-equivalence. Thus we may assume that each 2-cell of $X_{1}$ lies in some $p_{1}^{-1}\left(S_{\delta_{3}}(b)\right)$, and the attaching map for this 2-cell is null-homotopic in $p_{1}^{-1}\left(S_{\delta_{3}}(b)\right) \cap Y$. This enables us to use the 
idea of the proof of Corollary 3.3 to obtain a homotopy of each such 2-cell into $Y$, where this homotopy takes place in some $p^{-1}\left(S_{\delta_{4}+2 \gamma_{1}+2 \delta_{3}}(b)\right)$. Thus we can trade the 2-cells for 4-cells to obtain a relative $\mathrm{CW}$ complex $\left(X_{2}, Y\right)$ with cells of dimension $\geqslant 3$ and a $p_{1}^{-1}\left(\gamma_{2}\right)$-equivalence $f_{2}: X_{2} \rightarrow X_{1}$. Clearly $\gamma_{2}$ is small if $\delta_{4}+2 \gamma_{1}+2 \delta_{3}$ is small.

To see that $(X, Y)$ is $(\varepsilon, 2)$-connected let $\phi:\left(I^{2}, \partial I^{2}\right) \rightarrow(X, Y)$ be a map. Choose the subdivision of $X$ and adjust $\phi$ so that $\phi\left(I^{2}\right)$ lies in the 2-skeleton of $X$, say $\phi\left(I^{2}\right) \subset X^{2}$. We may choose $g_{1}: X \rightarrow X_{1}$ to be a $\gamma_{1}$-inverse of $f_{1}$ which is skeletapreserving. Similarly let $g_{2}: X_{1} \rightarrow X_{2}$ be chosen to preserve skeleta, but observe that we now have $X_{2}^{2}=Y$. Thus $g_{2} g_{1} \phi\left(I^{2}\right) \subset Y$, and we have a $p^{-1}(\varepsilon)$-homotopy

$$
\phi \simeq f_{1} f_{2} g_{2} g_{1} \phi \text { rel } \partial I^{2} \text {. }
$$

REMARKS. To see how the above argument adapts to the case in which $X$ is an ANR which is $\varepsilon$-finitely dominated for all $\varepsilon>0$, we first have to replace $X$ by a (possibly noncompact) relative $\mathrm{CW}$ complex. This is accomplished by using the mapping telescope construction with control, as explained in [2]. It asserts that for every $\gamma_{1}>0$ we can choose a relative $\mathrm{CW}$ complex $\left(X_{1}, Y\right)$ and a $\gamma_{1}$-equivalence $f_{1}: X_{1} \rightarrow X$ rel $Y$. Let $p_{1}=p f: X_{1} \rightarrow B$ be a controlling map for $X_{1}$, and it follows that $X_{1}$ is $\varepsilon_{1}$-finitely dominated, where $\varepsilon_{1}$ is a small number provided that $\gamma_{1}$ is small. By Proposition 5.1 (see Remark 1) we can attach a finite number of cells to $Y$ to obtain a finite relative CW complex $X_{2}$ and a map $f_{2}: X_{2} \rightarrow X_{1}$ which is a $\gamma_{2}$-domination rel $Y$ and which is $\left(\gamma_{2}, n+2\right)$-connected (with respect to the controlling map $\left.p_{1}: X_{1} \rightarrow B\right)$. Of course the size of $\gamma_{2}$ depends on the size of $\varepsilon_{1}$. If we let $X^{\prime}=X_{2}$ and $f^{\prime}=f_{1} f_{2}$, then it is easy to see that $f^{\prime}$ is $(\gamma, n+2)$-connected, where the size of $\gamma$ depends on the sizes of $\gamma_{1}$ and $\gamma_{2}$.

Let $p^{\prime}=p f^{\prime}: X^{\prime} \rightarrow B$ be a controlling map for $X^{\prime}$ and assume that $(X, Y)$ is homologically $\left(\delta_{2 i}, \delta_{2 i-1}, i\right)$-trivial and $Y \hookrightarrow X$ is a $\left(\delta_{1}, 1\right)$-equivalence. Again it is easy to see that $Y \hookrightarrow X^{\prime}$ is a $\left(\delta_{1}^{\prime}, 1\right)$-equivalence, where the size of $\delta_{1}^{\prime}$ depends on the sizes of $\gamma$ and $\delta_{1}$. It is our intention to show that $\left(X^{\prime}, Y\right)$ is homologically $\left(\delta_{2 i}^{\prime}, \delta_{2 i-1}, i\right)$-trivial, where the size of $\delta_{2 i}^{\prime}$ depends on the sizes of $\delta_{2 i}$ and $\gamma$. Once this has been done the above argument for the compact relative $\mathrm{CW}$ case applies to give $\left(X^{\prime}, Y\right)\left(\varepsilon^{\prime}, n\right)$-connected, and this in turn implies that $(X, Y)$ is $(\varepsilon, n)$-connected. We can subdivide the open set $\left(p^{\prime}\right)^{-1}\left(S_{\delta_{2}},(b)\right)$ of $X^{\prime}$ so that it is a relative CW complex. If $\left(p^{\prime}\right)^{-1}\left(S_{\delta_{21}},(b)\right)^{k}$ is its $k$-skeleton, then we only have to show that the following homomorphism is 0 :

$$
\begin{aligned}
H_{i}\left(\left(\left(p^{\prime}\right)^{-1}\left(S_{\delta_{2,-1}}(b)\right)^{n+1}\right) \sim,\left(\left(p^{\prime}\right)^{-1}\left(S_{\delta_{2,},}(b)\right) \cap Y\right)^{\sim}\right) \\
\rightarrow H_{i}\left(\left(p^{\prime}\right)^{-1}\left(S_{\delta_{2_{1}}}(b)\right)^{\sim},\left(\left(p^{\prime}\right)^{\prime}\left(S_{\delta_{i, 1}^{\prime}}(b)\right) \cap Y\right)^{\sim}\right) .
\end{aligned}
$$

First observe that $\left(X_{1}, Y\right)$ is homologically $\left(\delta_{2 i \mid \gamma_{1}}, \delta_{2 i}, i\right)$-trivial. Indeed the inclusion $\left(p f_{1}\right)^{-1}\left(S_{\delta_{2 i-1}}(b)\right) \hookrightarrow\left(p f_{1}\right)^{-1}\left(S_{\delta_{2 i}+\gamma_{1}}(b)\right)$ factors (up to homotopy) into

$$
\left(p f_{1}\right)^{-1}\left(S_{\delta_{2 i-1}}(b)\right) \stackrel{f_{1}}{\rightarrow} p^{-1}\left(S_{\delta_{2 i-1}}(b)\right) \hookrightarrow p^{-1}\left(S_{\delta_{2 i}}(b)\right) \stackrel{g_{1}}{\rightarrow}\left(. p f_{1}\right)^{-1}\left(S_{\delta_{2 i}+\gamma_{1}}(b)\right),
$$

where $g_{1}$ is a $\gamma_{1}$-inverse of $f_{1}$. By construction the map $f_{2}: X_{2} \rightarrow X_{1}$ is a $\gamma_{2}$-domination with inverse $g_{2}: X_{1} \rightarrow X_{2}$ for which the inclusion

$$
\left(p^{\prime}\right)^{-1}\left(S_{\delta_{2 i-1}}(b)\right)^{n+1} \hookrightarrow\left(p^{\prime}\right)^{-1}\left(S_{\delta_{2 i}^{\prime}}(b)\right)
$$


is homotopic to the composition

$$
\begin{aligned}
\left(p^{\prime}\right)^{-1}\left(S_{\delta_{2 i-1}}(b)\right)^{n+1} & \stackrel{f_{2}}{\rightarrow}\left(p f_{1}\right)^{-1}\left(S_{\delta_{2 i-1}}(b)\right) \hookrightarrow\left(p f_{1}\right)^{-1}\left(S_{\delta_{2 i}+\gamma_{1}}(b)\right) \\
& \stackrel{g_{2}}{\rightarrow}\left(p^{\prime}\right)^{-1}\left(S_{\delta_{2 i}+\gamma_{1}+\gamma_{2}}(b)\right)
\end{aligned}
$$

(see Remark 2 following Proposition 5.1). This is all we need.

4. Controlled matrices. The purpose of this section is to formulate the algebraic machinery which is needed for the proof of the Controlled Boundary Theorem that is given in $§ 6$. Here is a brief description of our problem. Let $(K, L)$ be a compact polyhedral pair, where $K$ is formed by attaching $k n$-cells to $L$ so that all have the same basepoint, i.e., the $i$ th one has attaching map $\phi_{i}:\left(\partial I^{n}, *\right) \rightarrow\left(L, l_{0}\right)$, for $l_{0}$ a fixed point of $L$. It is well known that for $n \geqslant 2$ (where the cells are trivially attached in case $n=2)$ the $Z \pi_{1}(L)$-module $\pi_{n}\left(K, L, l_{0}\right)$ is isomorphic to the direct sum of $k$-copies of $Z \pi_{1}(L)$. In this section we will treat a similar situation in which the $n$-cells of $K$ have different basepoints, and our problem will be to describe homotopy classes of maps $\left(I^{n}, \partial I^{n}\right) \rightarrow(K, L)$. To compound the problem these homotopy classes will be controlled over our parameter space $B$. In order to capture this full generality we have devised a notion of controlled matrices. The building blocks for these controlled matrices are the path groups as defined below.

First we treat the uncontrolled situation. For any space $X$ and $x, y \in X$ we define the path group $P[x, y]$ to be the free abelian group generated by the set of homotopy classes of paths from $x$ to $y$. If no such paths exist then we define $P[x, y]=0$. Thus $P[x, y]$ consists of all finite formal sums $\sum m_{r}\left[w_{r}\right]$, where $w_{r}$ is a path from $x$ to $y$. Now let $\hat{X}=X \cup e_{1}^{n} \cup \cdots \cup e_{k}^{n}, n \geqslant 2$, where $e_{j}^{n}$ is an $n$-cell attached to $X$ with attaching map $\phi_{j}:\left(\partial I^{n}, *\right) \rightarrow\left(X, y_{j}\right)$. Observe that for each $x \in X$ there exists a natural homomorphism $\alpha_{j}: P\left[x, y_{j}\right] \rightarrow \pi_{n}(\hat{X}, X, x)$ defined by sending $\sum m_{r}\left[w_{r}\right]$ to $\sum m_{r}\left[w_{r}^{-1} \hat{\phi}_{j}\right]$, where $\hat{\phi}_{j}:\left(I^{n}, \partial I^{n}, *\right) \rightarrow\left(\hat{X}, X, y_{j}\right)$ is a characteristic map for $e_{j}^{n}$ whose restriction to $\partial I^{n}$ is $\phi_{j}$ and $w_{r}^{-1} \hat{\phi}_{j}$ changes the basepoint by dragging the basepoint of $e_{j}^{n}$ along the path $w_{r}^{-1}$ from $y_{j}$ to $x$. Direct summing gives us a homomorphism

$$
\alpha=\sum \alpha_{j}: \sum P\left[x, y_{j}\right] \rightarrow \pi_{n}(\hat{X}, X, x) .
$$

We can easily give $\sum P\left[x, y_{j}\right]$ a $Z \pi_{1}(X, x)$-module structure so that $\alpha$ is a $Z \pi_{1}(X, x)$-module isomorphism. Then it easily follows from $[5, \S 8]$ that $\alpha$ is an isomorphism provided that the $e_{j}^{n}$ are trivially attached if $n=2$ (cf. Lemma 3.2 of this paper).

The above comments describe the classical situation, and we now introduce notation to describe the controlled case. Continuing the above notation let $p: X \rightarrow B$ be a controlling map, let $N_{\varepsilon}(x)=p^{-1}\left(S_{\varepsilon}(p(x))\right)$, and let

$$
\hat{N}_{\varepsilon}(x)=\cup\left\{e_{j}^{n} \mid \phi_{j}\left(\partial I^{n}\right) \subset N_{\varepsilon}(x)\right\} \cup N_{\varepsilon}(x) .
$$

We then define $P_{\varepsilon}[x, y]$ to be the $\varepsilon$-path group of all $\sum m_{r}\left[w_{r}\right]$, where $w_{r}$ is a path in $N_{\varepsilon}(x)$ from $x$ to $y$. As usual, $P_{\varepsilon}[x, y]=0$ if there are no such paths. In what follows 
we assume that $\operatorname{diam}\left(p \phi_{j}\left(\partial I^{n}\right)\right)<\varepsilon_{0}$, for all $j$. Then for every $\varepsilon>0$ there exists a natural homomorphism

$$
\alpha_{\varepsilon}: \sum P_{\varepsilon}\left[x, y_{j}\right] \rightarrow \pi_{n}\left(\hat{N}_{\varepsilon+\varepsilon_{0}}(x), N_{\varepsilon+\varepsilon_{0}}(x), x\right)
$$

which is defined in analogy with the homomorphism $\alpha$ described above. It is clear that the following diagram commutes for $\varepsilon \leqslant \delta$ :

$$
\begin{array}{ccc}
\sum P_{\varepsilon}\left[x, y_{j}\right] & \stackrel{\alpha_{\varepsilon}}{\rightarrow} & \pi_{n}\left(\hat{N}_{\varepsilon+\varepsilon_{0}}(x), N_{\varepsilon+\varepsilon_{0}}(x), x\right) \\
\downarrow & & \downarrow \\
\sum P_{\delta}\left[x, y_{j}\right] & \stackrel{\alpha_{\delta}}{\rightarrow} & \pi_{n}\left(\hat{N}_{\delta+\varepsilon_{0}}(x), N_{\delta+\varepsilon_{0}}(x), x\right)
\end{array}
$$

If $\beta$ is the inverse of the isomorphism $\alpha$, then for every $\varepsilon>0$ there is an $\varepsilon$-version of this homomorphism,

$$
\beta_{\varepsilon}: \pi_{n}\left(\hat{N}_{\varepsilon}(x), N_{\varepsilon}(x), x\right) \rightarrow \sum P_{\varepsilon}\left[x, y_{j}\right] .
$$

This satisfies the property that the following diagram commutes for $\varepsilon \leqslant \delta$ :

$$
\begin{array}{ccc}
\pi_{n}\left(\hat{N}_{\varepsilon}(x), N_{\varepsilon}(x), x\right) & \stackrel{\beta_{\varepsilon}}{\rightarrow} & \sum P_{\varepsilon}\left[x, y_{j}\right] \\
\downarrow & & \downarrow \\
\pi_{n}\left(\hat{N}_{\delta}(x), N_{\delta}(x), x\right) & \stackrel{\beta_{\delta}}{\rightarrow} & \sum P_{\delta}\left[x, y_{j}\right]
\end{array}
$$

Just as it was observed that $\alpha$ is an isomorphism, it similarly follows that $\beta_{\varepsilon+\varepsilon_{10}} \alpha_{\varepsilon}$ and $\alpha_{\varepsilon} \beta_{\varepsilon}$ are inclusion-induced, for all $\varepsilon>0$.

By a path matrix $A=\left(a_{i j}\right)$ on $X$ we mean a finite matrix whose $i$ th row corresponds to $x_{i} \in X$ and whose $j$ th column corresponds to $y_{j} \in X$ (with possible repetitions), and $a_{i j} \in P\left[x_{i}, y_{j}\right]$. An $\varepsilon$-path matrix is essentially the same thing except that $a_{i j} \in P_{\varepsilon}\left[x_{i}, y_{j}\right]$. The domain of $A$ is the ordered tuple $\left(x_{i}\right)$, and the range of $A$ is the ordered tuple $\left(y_{j}\right)$, written $A:\left(x_{i}\right) \rightarrow\left(y_{j}\right)$. If $A=\left(a_{i j}\right):\left(x_{i}\right) \rightarrow\left(y_{j}\right)$ is an $\varepsilon$-path matrix and $B=\left(b_{j k}\right):\left(y_{j}\right) \rightarrow\left(z_{k}\right)$ is a $\delta$-path matrix, then $A B:\left(x_{i}\right) \rightarrow\left(z_{k}\right)$ is the $(\varepsilon+\delta)$-path matrix $\left(\sum_{j} a_{i j} * b_{j k}\right)$. Here the symbol $*$ is used to denote the composition of homotopy classes of paths.

In the sequel we will use $\varepsilon$-path matrices to describe controlled homotopy classes as was discussed earlier. The following remarks are intended to make it clear how $\varepsilon$-path matrices will be used. Just as above $p: X \rightarrow B$ will be a controlling map and $(\hat{X}, X)$ will be a relative $\mathrm{CW}$ complex so that $\operatorname{diam}\left(p \phi_{j}\left(\partial I^{n}\right)\right)<\varepsilon_{0}$, where $\phi_{j}:\left(\partial I^{n}, *\right) \rightarrow\left(X, y_{j}\right)$ is the attaching map for $e_{j}^{n}$. Let $\left(x_{i}\right)$ be any finite ordered tuple of points of $X$.

1. If $A:\left(x_{i}\right) \rightarrow\left(y_{j}\right)$ is an $\varepsilon$-path matrix, then for each $i$ the $i$ th row of $A$ determines a map $\psi_{i}:\left(I^{n}, \partial I^{n}, *\right) \rightarrow\left(\hat{X}, X, x_{i}\right)$ so that $\psi_{i}\left(I^{n}\right) \subset \hat{N}_{\varepsilon+\varepsilon_{0}}\left(x_{i}\right) . \psi_{i}$ arises from the homomorphism $\alpha_{\varepsilon}$.

2. If $\psi_{i}:\left(I^{n}, \partial I^{n}, *\right) \rightarrow\left(\hat{X}, X, x_{i}\right)$ is a map so that $\psi_{i}\left(I^{n}\right) \subset \hat{N}_{\varepsilon}\left(x_{i}\right)$, then there is an $\varepsilon$-path matrix $A:\left(x_{i}\right) \rightarrow\left(y_{j}\right)$ whose $i$ th row is determined by $\psi_{i}$. The elements of this row arise from the homomorphism $\beta_{\varepsilon}$. 
5. Controlled Wall theory. The purpose of this section is to establish controlled versions of some aspects of Wall's finiteness obstruction [10]. As in $\$ 4$ we will have to deal with the problem of multiple basepoints, so we will be using the controlled matrices which were defined there. We will first establish the following result which treats the notion of controlled cellular surgery on a map. For notation let $K$ and $L$ be polyhedra such that $K$ is compact and let $p: L \rightarrow B$ be a controlling map to our parameter space such that $L$ is $\varepsilon$-finitely dominated, for all $\varepsilon>0$.

Proposition 5.1. For every $\varepsilon>0$ and $n \geqslant 0$ there exists a $\delta>0$ so that if $f: K \rightarrow L$ is $(\delta, n-1)$-connected, then by attaching a finite number of $n$-cells to $K$ we can form $\tilde{K}$ and extend $f$ to a map $\tilde{f}: \tilde{K} \rightarrow L$ which is $(\varepsilon, n)$-connected. Moreover, $\delta$ depends only on $\varepsilon$ and $n$.

Proof. By the standard mapping cylinder trick we may assume that $K$ is a subpolyhedron of $L$ and $f$ is the inclusion $K \hookrightarrow L$. Choose a fine subdivisiion of $L$ and let $L^{k}$ be its $k$-skeleton. By using the $(\delta, n-1)$-connectivity of $K \hookrightarrow L$ we can inductively work through the skeleta of $L^{n-1}$ to construct a map $\phi: K \cup L^{n-1} \rightarrow K$ for which $\phi \mid K=$ id and $\phi$ is $p^{-1}\left(\delta^{\prime}\right)$-homotopic to $K \cup L^{n-1} \hookrightarrow L$ rel $K$, where $\delta^{\prime}$ is small if $\delta$ is small. Since $L$ is $\varepsilon$-finitely dominated, for all $\varepsilon>0$, we can find a compact subpolyhedron $J$ of $L$ containing $K$ for which there is a $p^{-1}(\delta)$-homotopy of $L$ into $J$ rel $K$. In the following construction of $\tilde{f}: \tilde{K} \rightarrow L$ it will be clear that if $\delta$ and $\delta^{\prime}$ are small enough, then the desired $\varepsilon$-control on the $n$-connectivity of $\tilde{f}$ is achieved.

We define $\tilde{K}$ to be the quotient space $\left(K \cup J^{n}\right) / \sim$, where $\sim$ is the equivalence relation generated by $x \sim \phi(x)$, for all $x \in J^{n-1}$. Let $q: K \cup J^{n} \rightarrow \tilde{K}$ be the resulting quotient map. To define $\tilde{f}: \tilde{K} \rightarrow L$ choose any $n$-simplex $\Delta^{n} \subset J^{n}$ and note that $q\left(\Delta^{n}\right)$ is a typical $n$-cell in $\tilde{K}$ which is attached to $K$. Since we want $\tilde{f} \mid K=$ id we will only have to concentrate on the definition of $\tilde{f}$ on $q\left(\Delta^{n}\right)$. For this it will be convenient to define $\Delta^{n}=[-1,1]^{n}$ and, more generally, $\Delta_{t}^{n}=[-t, t]^{n}$. On $q\left(\Delta_{1 / 2}^{n}\right)$ we define $\tilde{f}$ to be $\gamma q^{-1}$, where $\gamma: \Delta_{1 / 2}^{n} \rightarrow \Delta^{n}$ is a radially-defined homeomorphism. On $q\left(\partial \Delta_{t}^{n}\right), \frac{1}{2} \leqslant t \leqslant 1$, we define $\tilde{f}$ to be $\gamma_{t} q^{-1}$ followed by the $(2 t-1)$-level of the homotopy id $\simeq \phi$, where $\gamma_{t}: \partial \Delta_{t}^{n} \rightarrow \partial \Delta^{n}$ is a radially-defined homeomorphism.

Finally we have to show that $\tilde{f}: \tilde{K} \rightarrow L$ is $(\varepsilon, n)$-connected. So let $g: I^{k} \rightarrow L$, $g_{0}: \partial I^{k} \rightarrow \tilde{K}$ be maps for which $\tilde{f} g_{0}=g \mid \partial I^{k}$, for $k \leqslant n$. By general positioning we may assume that $g_{0}\left(\partial I^{k}\right) \subset K$. We want a map $\tilde{g}: I^{k} \rightarrow \tilde{K}$ for which $\tilde{g} \mid \partial I^{k}=g_{0}$ and $\tilde{f} \tilde{g} \simeq g$ rel $\partial I^{k}$ via a $p^{-1}(\varepsilon)$-homotopy. To do this we first deform $g$ so that it is into $J$, and then by general positioning we may assume that it is into $J^{n}$. Finally composing with $q: K \cup J^{n} \rightarrow \tilde{K}$ gives us our desired map $\tilde{g}: I^{k} \rightarrow \tilde{K}$. It is easy to see that $\tilde{f} \tilde{g} \simeq g$ rel $\partial I^{k}$ via a $p^{-1}(\varepsilon)$-homotopy (for appropriately small choices of $\delta$ and $\delta^{\prime}$ ).

RemarKs. 1. The above proof easily applies to the more general setting as encountered in the proof of Theorem 3.1. Suppose we are given a relative $C W$ complex $(L, K), K$ compact, and a map $p: L \rightarrow B$. For every $\varepsilon>0$ and $n \geqslant 0$ there exists a $\delta>0$ (depending only on $\varepsilon$ and $n$ ) so that if $L$ is $\delta$. finitely dominated, then there exists a compact relative $\mathrm{CW}$ complex $(\tilde{K}, K)$ and a map $f: \tilde{K} \rightarrow L$ which is $(\varepsilon, n)$-connected and which is an $\varepsilon$-domination rel $K$. To prove this let $K_{0} \supset K$ be a 
compact subcomplex of $L$ for which $K_{0} \hookrightarrow L$ is a $\delta$-domination rel $K$. Thus $K_{0} \hookrightarrow L$ is $(\delta, 0)$-connected, so we can apply the above proof $n$ times to the inclusion $K_{0} \hookrightarrow L$ to extend it to our desired $f: \tilde{K} \rightarrow L$.

2. Also in the above general setting it follows that if $\left(J^{n-1}, K\right)$ is a relative $\mathrm{CW}$ complex and $\phi: J^{n-1} \rightarrow \tilde{K}$ is a map such that $\phi \mid K=$ id, then there exists a $(p f)^{-1}\left(\varepsilon_{1}\right)$-homotopy $\phi \simeq g f \phi$ rel $K$, where $g: L \rightarrow \tilde{K}$ is a right $\varepsilon$-inverse of $f$ and $\varepsilon_{1}$ is small if $\varepsilon$ is small. This is done by using the $(\varepsilon, n)$-connectivity of $f$ to lift the $p^{-1}(\varepsilon)$-homotopy $f \phi \simeq(f g) f \phi$ from $L$ to $\tilde{K}$.

We now establish a result which says that in certain situations cells can be traded down to lower-dimensional cells with control in the parameter space $B$. For notation let $(J, K)$ and $(L, J)$ be compact relative $C W$ complexes, where $J=K \cup\{(n+1)$ cells $\}$ and $L=J \cup\{(n+2)$-cell $\}, n \geqslant 2$. Also let $p: L \rightarrow B$ be a controlling map to our parameter space.

Lemma 5.2. For every $\varepsilon>0$ there exists a $\delta>0$ so that if $J \hookrightarrow L$ is a $\delta$-domination rel $K$ and the images of the $(n+1)$ - and $(n+2)$-cells of $L$ have diameters $<\delta$ in $B$, then there exist compact relative $\mathrm{CW}$ complexes $\left(J^{\prime}, K\right)$ and $\left(L^{\prime}, J^{\prime}\right)$, where $J^{\prime}=K^{\prime}$ $\cup\{n$-cells $\}$ and $L^{\prime}=J^{\prime} \cup\{(n+1)$-cells $\}$, and an $\varepsilon$-equivalence $L^{\prime} \simeq L$ rel $K$. Moreover, $\delta$ depends only on $\varepsilon$.

Proof. Let the $(n+1)$-cells of $J$ have characteristic maps $\phi_{i}:\left(I^{n+1}, \partial I^{n+1}, *\right) \rightarrow$ $\left(J, K, x_{i}\right), 1 \leqslant i \leqslant k$, and let the $(n+2)$-cells of $L$ have characteristic maps $\psi_{j}:\left(I^{n+2}, \partial I^{n+2}, *\right) \rightarrow\left(L, J, y_{j}\right), 1 \leqslant j \leqslant l$. We may choose $y_{j} \in K$ since the cells have small diameters in $B$. The definition of $J^{\prime}$ is easy. It is simply formed by attaching $l n$-cells to $K$, where the $j$ th one is trivially attached at $y_{j} \in K$. The definition of $L^{\prime}$ is a little harder. There will be $k(n+1)$-cells of $L^{\prime}$-one for each $(n+1)$-cell of $J$. The $i$ th $(n+1)$-cell will be based at $x_{i}$, and the attaching map for this cell will be a map $\theta_{i}:\left(I^{n+1}, *\right) \rightarrow\left(J^{\prime}, x_{i}\right)$ as described below.

Our hypotheses give us a map $g: L \rightarrow J$ which satisfies $g \simeq$ id rel $K$ via a $p^{-1}(\delta)$ homotopy. So for each $i$ the homotopy exact sequence of $(L, J, K)$ (based at $x_{i}$ ) gives us a split short exact sequence,

$$
0 \rightarrow \pi_{n+2}(L, J) \underset{r_{i}}{\rightarrow} \pi_{n+1}(J, K) \underset{u_{i}}{\rightarrow} \pi_{n+1}(L, K) \rightarrow 0 .
$$

The splitting map $u_{i}$ is induced by $g$, and the splitting map $r_{i}$ enjoys the property that $r_{i}\left(\left[\phi_{i}\right]\right)=\left[\tilde{\phi}_{i}\right]$, where $\tilde{\phi}_{i}:\left(I^{n+2}, \partial I^{n+2}, *\right) \rightarrow\left(L, J, x_{i}\right)$ is a map for which diam $p \tilde{\phi}_{i}\left(I^{n+2}\right)$ is small provided that $\delta$ is small. The entire collection of maps $\left\{\tilde{\phi}_{i}\right\}$ determines a $\delta^{\prime}$-path matrix $R:\left(x_{i}\right) \rightarrow\left(y_{j}\right)$. The $i$ th row of $R$ determines a map $\theta_{i}^{\prime}:\left(\partial I^{n+1}, *\right) \rightarrow\left(J^{\prime}, x_{i}\right)$ (since the cells of $J^{\prime}$ are trivially attached). We also have a map $\phi_{i} \mid \partial I^{n+1}:\left(\partial I^{n+1}, *\right) \rightarrow\left(K, x_{i}\right) \subset\left(J^{\prime}, x_{i}\right)$, and so we can form a map $\theta_{i}:\left(\partial I^{n+1}, *\right) \rightarrow\left(J^{\prime}, x_{i}\right)$ for which $\left[\theta_{i}\right]=\left[\theta_{i}^{\prime}\right]+\left[\phi_{i} \mid \partial I^{n+1}\right]$. This map is used as an attaching map to attach the $i$ th $(n+1)$-cell of $L^{\prime}$ to $J^{\prime}$, thus completing the definition of $L^{\prime}$. Observe that $\delta^{\prime}$ can be chosen small provided that $\delta$ is also chosen small. In what follows below it will be convenient to write $J=K \cup d_{1} \cup \cdots \cup d_{k}$ and $L=J \cup e_{1} \cup \cdots \cup e_{l}$, where $d_{i}$ has characteristic map $\phi_{i}$ and $e_{j}$ has characteristic map $\psi_{j}$. Similarly let $J^{\prime}=K \cup e_{1}^{\prime} \cup \cdots \cup e_{l}^{\prime}$ and $L^{\prime}=J^{\prime} \cup d_{1}^{\prime} \cup \cdots \cup d_{k}^{\prime}$. 
Our method of showing that $L^{\prime}$ is homotopy equivalent to $L$ rel $K$ is to trade the $n$-cells of $J^{\prime}$ for $(n+2)$-cells, thus forming $\mathrm{CW}$ complexes

$$
\begin{aligned}
& J^{\prime \prime}=K \cup\{(n+1) \text {-cells }\}=K \cup d_{1}^{\prime \prime} \cup \cdots \cup d_{k}^{\prime \prime}, \\
& L^{\prime \prime}=J^{\prime \prime} \cup\{(n+2) \text {-cells }\}=J^{\prime \prime} \cup e_{1}^{\prime \prime} \cup \cdots \cup e_{l}^{\prime \prime} .
\end{aligned}
$$

In order that $L^{\prime \prime}$ be homotopy equivalent to $L$ with $\varepsilon$-control in $B$ it suffices that the following be true (cf. [5, 7.1]):

(1) the attaching maps of $d_{i}$ and $d_{i}^{\prime \prime}$ are homotopic (small homotopy in $B$ ),

(2) the attaching maps of $e_{j}$ and $e_{j}^{\prime \prime}$ are homotopic (small homotopy in $B$ ).

Our desired controlled homotopy equivalence $L^{\prime} \simeq L$ is then a composition $L^{\prime} \simeq L^{\prime \prime} \simeq L$, where the first homotopy equivalence easily results from the cell-trading process.

To carry out the cell-trading process we will have to obtain a homotopy of each $e_{j}^{\prime}$ into $K$, with the homotopy taking place in $L^{\prime}$. This is done by finding an element of $\pi_{n+1}\left(L^{\prime}, J^{\prime}, y_{j}\right)$ whose image in $\pi_{n}\left(J^{\prime}, K, y_{j}\right)$ under the boundary homomorphism $\partial_{j}^{\prime}: \pi_{n+1}\left(L^{\prime}, J^{\prime}, y_{j}\right) \rightarrow \pi_{n}\left(J^{\prime}, K, y_{j}\right)$ is the homotopy class of $e_{j}$. This element of $\pi_{n+1}\left(L^{\prime}, J^{\prime}\right)$ then provides a homotopy of $e_{j}^{\prime}$ into $K$ as desired. Of course all of this must be done with small control in $B$. Our first step is to obtain a path matrix representing the boundary homomorphisms

$$
\partial_{j}: \pi_{n+2}\left(L, J, y_{j}\right) \rightarrow \pi_{n+1}\left(J, K, y_{j}\right) .
$$

For each $j$ the restriction $\psi_{j} \mid:\left(\partial I^{n+2}, *\right) \rightarrow\left(J, y_{j}\right)$ may be regarded as a map of $\left(I^{n+1}, \partial I^{n+1}, *\right)$ into $\left(J, K, y_{j}\right)$, and so the entire collection of maps determines a $2 \delta$-path matrix $\partial:\left(y_{j}\right) \rightarrow\left(x_{i}\right)$. The next step is to interpret the $j$ th row of $\partial$ as a map $\sigma_{j}:\left(I^{n+1}, \partial I^{n+1}, *\right) \rightarrow\left(L^{\prime}, J^{\prime}, y_{j}\right)$, thus given $\left[\sigma_{j}\right] \in \pi_{n+1}\left(L^{\prime}, J^{\prime}, y_{j}\right)$. Now observe that $\partial_{j}^{\prime}\left(\left[\sigma_{j}\right]\right)$ is determined by the $j$ th row of the product matrix $\partial R$, and this is the identity matrix by the manner in which the $r_{i}$ were chosen. This means that $\partial_{j}^{\prime}\left(\left[\sigma_{j}\right]\right)$ is the homotopy class of $e_{j}^{\prime}$, and so $\sigma_{j}$ can be used to deform $e_{j}^{\prime}$ into $K$. Therefore the cell-trading process can be carried out to trade the $e_{j}^{\prime}$ for $(n+2)$-cells $e_{j}^{\prime \prime}$. At the same time the $d_{i}^{\prime}$ are deformed to new $(n+1)$-cells $d_{i}^{\prime \prime}$.

Since the $e_{j}^{\prime}$ are trivially attached the $R$-component of the attaching map of a typical $d_{i}^{\prime}$ disappears when it is deformed to $d_{i}^{\prime \prime}$, and so $d_{i}^{\prime \prime}$ is attached to $K$ according to the map $\phi_{i} \mid \partial I^{n+1}$. Also the homotopy used to deform a typical $e_{j}^{\prime}$ into $K$ becomes the attaching map of $e_{j}^{\prime \prime}$, and so the $e_{j}^{\prime \prime}$ are attached to $J^{\prime \prime}$ according to the matrix $\partial$. This is in agreement with the situation for $J$ and $L$.

We are now ready for the main result of this section. Here is a brief description of our problem. Let ( $L, J, K$ ) be a polyhedral triple (all connected), where $J$ and $K$ are compact and $J$ is formed by attaching $n$-cells to $K, n \geqslant 2$. Assume further that $(L, J)$ is $n$-connected and the inclusion $J \hookrightarrow L$ is a domination rel $K$. One easily shows that the inclusion-induced homomorphism of $Z \pi_{1}(K)$-modules, $\pi_{n}(J, K) \rightarrow$ $\pi_{n}(L, K)$, is split, so $\pi_{n}(J, K) \approx \pi_{n+1}(L, J) \oplus \pi_{n}(L, K)$. It follows from the results of [10] that if $L$ is homotopy equivalent to a finite complex, then $\pi_{n}(L, K)$ is stably free. This quickly implies that there are free f.g. $Z \pi_{1}(K)$-modules $F_{1}, F_{2}$ and an isomorphism

$$
\pi_{n}(J, K) \oplus F_{1} \oplus F_{2} \approx \pi_{n}(J, K) \oplus F_{1} \oplus F_{2}
$$


which takes $\pi_{n}(J, K) \oplus F_{1}$ onto $\pi_{n}(L, K) \oplus F_{2}$ and which takes $F_{2}$ onto $\pi_{n+1}(L, J)$ $\oplus F_{1}$. The next result establishes a controlled version of this situation.

For notation let $(J, K)$ and $(L, K)$ be relative CW complexes, where $J$ is compact and it is formed by attaching $n$-cells to $K, n \geqslant 2$, with basepoints $x_{1}, x_{2}, \ldots, x_{r}$. Also let $p: L \rightarrow B$ be a controlling map to our parameter space and let $f: J \rightarrow L$, $f \mid K=$ id, be a map which is $(\delta, n)$-connected and which is a $\delta$-domination rel $K$. If $g: L \rightarrow J$ is a $\delta$-inverse of $f$ rel $K$, then we get a map $g f: J \rightarrow J$. Let $p f: J \rightarrow B$ be a controlling map for $J$ and assume that the diameters of the images of the $n$-cells of $J$ in $B$ are less than $\delta$. According to the notation of $\$ 4$ the images of the $n$-cells of $J$ under the $g f$ yield a $2 \delta$-path matrix $A:\left(x_{i}\right) \rightarrow\left(x_{i}\right)$. If $I:\left(x_{i}\right) \rightarrow\left(x_{i}\right)$ is the identity path matrix, then we can also form the $2 \delta$-path matrix $I-A:\left(x_{i}\right) \rightarrow\left(x_{i}\right)$, where $-A$ is formed by multiplying each element of $A$ by -1 and addition is just addition in the path groups.

THEOREM 5.3. Let $\varepsilon>0$ and an integer $m>0$ be given. The number $\delta$ mentioned above can be chosen small enough so that if there exists a compact relative $\mathrm{CW}$ complex $(P, K)$ of dimension $\leqslant m$ and a $\delta$-equivalence $P \simeq L \mathrm{rel} K$, then there exists tuples $\left(y_{j}\right)$ and $\left(z_{k}\right)$ on $K$ and an e-path isomorphism $B:\left(\left(x_{i}\right),\left(y_{j}\right),\left(z_{k}\right)\right) \rightarrow$ $\left(\left(x_{i}\right),\left(y_{j}\right),\left(z_{k}\right)\right)$ for which

$$
\left(\begin{array}{lll}
A & 0 & 0 \\
0 & 0 & 0 \\
0 & 0 & I
\end{array}\right) B\left(\begin{array}{lll}
0 & 0 & 0 \\
0 & 0 & 0 \\
0 & 0 & I
\end{array}\right)=0
$$

and

$$
\left(\begin{array}{ccc}
I-A & 0 & 0 \\
0 & I & 0 \\
0 & 0 & 0
\end{array}\right) B\left(\begin{array}{ccc}
I & 0 & 0 \\
0 & I & 0 \\
0 & 0 & 0
\end{array}\right)=0
$$

REMARKs. The number $\delta$ in the above statement depends only on $\varepsilon, n$ and $m$. Also the rows and columns in the above matrices are arranged in blocks with the order being $\left(x_{i}\right),\left(y_{j}\right)$ and $\left(z_{k}\right)$.

Proof. By a mapping cylinder construction we may assume that $J \subset P$ so that $(P, J)$ is a relative CW complex. Since we have a $\delta$-equivalence $P \simeq L$ we may assume that $J \hookrightarrow P$ is a $\delta$-domination rel $K$ and $(P, J)$ is $(\delta, n)$-connected, i.e., $J \hookrightarrow P$ is $(\delta, n)$-connected. Thus we can use cell-trading to further assume that $P-J$ has no cells of dimension $<n$ (cf. Lemma 3.4). (Our controlling map $P \rightarrow B$ is the composition $P \simeq L \stackrel{p}{\rightarrow} B$.) Let $P^{i}$ denote the $i$-skeleton of $P$, i.e., $P^{i}$ is the union of $J$ and all cells of $P-J$ of dimension $\leqslant i$. By the controlled homotopy extension theorem [4, §2] we observe that if $i \geqslant n$, then $P^{i+1} \hookrightarrow P$ is a $\delta^{\prime}$-domination rel $P^{i}$, where $\delta^{\prime}$ is small if $\delta$ is small. Thus by inductive use of Lemma 5.2 the cells of $P$ can be traded down. This means that we may additionally assume $m=n+1$. So in this simplified model $P$ consists of $J$ with a finite number of $n$-cells attached to $J$, and then a finite number of $(n+1)$-cells attached to this. Since $(P, J)$ is $(\delta, n)$-connected these additional $n$-cells of $P$ may be taken to be trivially attached to $K$. Let $J_{1}$ 
be the union of $K$ and these trivially attached $n$-cells, say attached at $\left(y_{j}\right)$ in $K$. Let $\left(z_{k}\right)$ in $K$ be the basepoints for the remaining $(n+1)$-cells of $P$. Here is a picture of $P$.

\begin{tabular}{|c|c|}
\hline$J_{1}\{n$-cells $\}$ & \multirow{2}{*}{$n+1$} -cells \\
\cline { 1 - 1 }$J\{n$-cells $\}$ & \\
\hline
\end{tabular}

Now for some notation. If $h: P \rightarrow L$ is the given $\delta$-equivalence, then $p h: P \rightarrow B$ is our controlling map to $B$ and we may assume that diam $p h(e)$ is small, for each cell $e$ of $P$. We are given a map $g^{\prime}: P \rightarrow J$ for which $g^{\prime} \simeq$ id rel $K$, where this is a $(p h)^{-1}(\delta)$-homotopy. The restriction $g^{\prime} \mid: J \rightarrow J$ is the map that produces our given matrix $A$. Since $J \cup J_{1} \hookrightarrow P$ is a $\delta$-domination rel $K$ we are in a situation similar to that which arose in the proof of Lemma 5.2. So if $\partial:\left(z_{k}\right) \rightarrow\left(\left(x_{i}\right),\left(y_{i}\right)\right)$ is the $2 \delta$-path matrix determined by the attaching maps for the $(n+1)$-cells of $P$, then for $\delta^{\prime}$ small there is a $\delta^{\prime}$-path matrix $R:\left(\left(x_{i}\right),\left(y_{j}\right)\right) \rightarrow\left(z_{k}\right)$ for which $\partial R=I_{\left(z_{k}\right)}$. It is also easily seen that $R \partial=(I-A) \oplus I_{(y,)}$. Now define our desired matrix $B:\left(\left(x_{i}\right),\left(y_{j}\right),\left(z_{k}\right)\right) \rightarrow\left(\left(x_{i}\right),\left(y_{j}\right),\left(z_{k}\right)\right)$ by

$$
B=\left(\begin{array}{ccc}
A & 0 & (I-A) R \\
0 & 0 & R \\
\partial_{x} & \partial_{y} & 0
\end{array}\right) .
$$

This has an inverse,

$$
B^{-1}=\left(\begin{array}{ccc}
A & 0 & R \\
0 & 0 & R \\
\partial_{x} & \partial_{y} & 0
\end{array}\right),
$$

so it is an isomorphism. If one observes that $A^{2}=A$ and $(I-A)^{2}=I-A$, then it is easy to check that $B$ fulfills our requirements.

6. Proof of the controlled boundary theorem. We are given $p: M^{m} \rightarrow B$ which is tame at $\infty$, 1-movable at $\infty$, and nice at $\infty$. Our strategy for putting a controlled boundary on $M$ is similar to that of Siebenmann [8] or Quinn [7]. The first step is to show that for every sequence $\varepsilon_{1}>\varepsilon_{2}>\cdots, \lim \varepsilon_{i}=0$, there are neighborhoods $N_{1} \supset \supset N_{2} \supset \supset \cdots$ of $\infty, \cap N_{i}=\varnothing$, so that $\partial N_{i} \hookrightarrow N_{i}-\stackrel{\circ}{i+1}_{i}$ and $\partial N_{i+1} \hookrightarrow N_{i}-$ $\stackrel{\circ}{i+1}_{i}$ are $\left(\varepsilon_{i}, 1\right)$-equivalences. (Notation such as this will be used throughout this section; it means that each $N_{i}$ is a clean submanifold of $M$ for which $M-\stackrel{\circ}{i}_{i}$ is compact and $N_{i+1} \subset \stackrel{\circ}{i}_{i}$.) This first step is just a controlled version of the corresponding step in Siebenmann [8] where small 1-neighborhoods of $\infty$ are constructed. The next step is to show that the sequence $\left\{N_{i}\right\}$ can be chosen to additionally satisfy the property that $\left(N_{i}, \partial N_{i}\right)$ is $\left(\varepsilon_{i}, m-3\right)$-connected, i.e., $\partial N_{i} \hookrightarrow N_{i}$ is $\left(\varepsilon_{i}, m-3\right)$ connected. These two steps only use the tameness at $\infty$ and the 1-movability at $\infty$. The third step is to use the niceness at $\infty$ to rechoose the $N_{i}$ so that $\left(N_{i}, \partial N_{i}\right)$ is $\left(\varepsilon_{i}, m-2\right)$-connected. Finally the last step is to explain why the existence of these 
( $m-2$ )-neighborhoods implies that $M$ admits a controlled boundary. There are many parts of the argument of Quinn [7] which apply here even though we do not assume that $B$ is locally simply connected and we do not assume that $\pi_{1}$ is locally constant. We shall take advantage of this similarity to significantly simplify some of the steps below.

I. 1-Neighborhoods of $\infty$. Given any $\varepsilon>0$ we will first show how to construct a neighborhood $N$ of $\infty$ so that $\partial N \hookrightarrow N$ is an $(\varepsilon, 1)$-equivalence. In order to do this we only need the tameness of $p$ at $\infty$. Let $N^{\prime} \subset M$ by any neighborhood of $\infty$. By repeated application of Proposition 5.1 it follows that for every $\varepsilon>0$ we can attach a finite number of 0 -, 1 - and 2-cells to $\partial N^{\prime}$, thus forming a $\mathrm{CW}$ complex $K$, and extend $\partial N^{\prime} \hookrightarrow N^{\prime}$ to a map $f: K \rightarrow N^{\prime}$ which is $(\varepsilon, 2)$-connected. Since $m \geqslant 5$ we can embed $K$ in $N^{\prime}$ rel $\partial N^{\prime}$ and take $f$ to be the inclusion $K \hookrightarrow N^{\prime}$. Let $R \subset M$ be a regular neighborhood of $\overline{M-N^{\prime}} \cup K$ and let $N=\overline{M-R}$. If $R$ is sufficiently close to $\overline{M-N^{\prime}} \cup K$ and $m \geqslant 5$, then it is easy to check that $\partial N \hookrightarrow N$ is an $(\varepsilon, 1)$-equivalence simply by using the fact that $K \hookrightarrow N^{\prime}$ is $(\varepsilon, 2)$-connected along with general positioning. Observe that if $m \geqslant 6$, then this argument shows that $\partial N \hookrightarrow N$ is $(\varepsilon, 2)$-connected.

The next step is to perform a similar maneuver on the left, and for this we only need the 1-movability of $p$ at $\infty$. Let $C \subset D$ be compacta in $M$ and let $N \subset M-D$ be a neighborhood of $\infty$. We say that $f: K \rightarrow \overline{M-N}-D$ is an $(\varepsilon, n)$-equivalence (or is $(\varepsilon, n)$-connected) in $\overline{M-N}-C$ provided that the lifting property which appears in the related definitions of $\S 2$ is satisfied, but the homotopy $f \tilde{\phi} \simeq \phi$ is allowed to take place in $\overline{M-N}-C$. If we are to perform our maneuver on the left as above we can no longer directly apply the statement of Proposition 5.1, however the proof still applies. Using the proof of Proposition 5.1 it follows that for every $\varepsilon>0$ and compactum $C \subset M$, there exists a compactum $D \supset C$ which satisfies the following property: for every neighborhood $N^{\prime} \subset M-D$ of $\infty$ which is sufficiently close to $\infty$, we can attach a finite number of $0-, 1-$, and 2-cells to $\partial N^{\prime}$ to form $K$ so that the inclusion $\partial N^{\prime} \hookrightarrow \overline{M-N^{\prime}}-D$ extends to a map $f: K \rightarrow \overline{M-N^{\prime}}-D$ which is an $(\varepsilon, 1)$-equivalence in $\overline{M-N^{\prime}}-C$. Since $m \geqslant 5$ we may take $K$ to be in $\overline{M-N^{\prime}}-D$ so that $f$ is the inclusion.

Now let $N$ be a regular neighborhood of $N^{\prime} \cup K$. If $N$ is sufficiently close to $N^{\prime} \cup K$ it follows that $\partial N \hookrightarrow \overline{M-N}-D$ is an $(\varepsilon, 1)$-equivalence in $\overline{M-N}-C$. Note that if $N^{\prime}$ additionally satisfied the property that $\partial N^{\prime} \hookrightarrow N^{\prime}$ is an $(\varepsilon, 1)$-equivalence, then $K \hookrightarrow N^{\prime} \cup K$ is still an $\left(\varepsilon^{\prime}, 1\right)$-equivalence (by definition), where $\varepsilon^{\prime}$ is small if $\varepsilon$ is small. So by a general position argument it follows that $\partial N \hookrightarrow N$ is an $\left(\varepsilon^{\prime}, 1\right)$-equivalence. Thus we have shown that for every $\varepsilon>0$ and compactum $C \subset M$ there exists a compactum $D \supset C$ which satisfies the following property: for every compactum $E \supset D$ there exists a neighborhood $N \subset M-E$ of $\infty$ such that $\partial N \hookrightarrow N$ is an $(\varepsilon, 1)$-equivalence and $\partial N \hookrightarrow \overline{M-N}-D$ is an $(\varepsilon, 1)$-equivalence in $\overline{M-N}-C$.

We are now ready for the last part of the argument. We want to prove that for every $\varepsilon_{1}>\varepsilon_{2}>\cdots, \lim \varepsilon_{i}=0$, there are neighborhoods $N_{1} \supset \supset N_{2} \supset \supset \cdots$ of $\infty$, $\cap N_{i}=\varnothing$, so that $\partial N_{i} \hookrightarrow N_{i}-\stackrel{\circ}{N}_{i+1}$ and $\partial N_{i+1} \hookrightarrow N_{i}-\dot{N}_{i+1}$ are $\left(\varepsilon_{i}, 1\right)$-equivalences. We will only give the details for the construction of $N_{1}$ and $N_{2}$, as this will indicate how the construction goes in general. Choose any $\varepsilon_{1}^{\prime}, \varepsilon_{1}^{\prime \prime}>0$ and start by choosing $N_{1}$ 
to be a neighborhood of $\infty$ for which $\partial N_{1} \hookrightarrow N_{1}$ is an $\left(\varepsilon_{1}^{\prime}, 1\right)$-equivalence and $\partial N_{1}$ $\hookrightarrow \overline{M-N_{1}}-D$ is an $\left(\varepsilon_{1}^{\prime}, 1\right)$-equivalence in $\overline{M-N_{1}}-C$, for some compacta $C \subset D$ $\subset M-N_{1}$. Now choose another neighborhood $N_{2} \subset \subset N_{1}$ of $\infty$ so that $\partial N_{2} \hookrightarrow N_{2}$ is an $\left(\varepsilon_{1}^{\prime \prime}, 1\right)$-equivalence and $\partial N_{2} \hookrightarrow N_{1}-\stackrel{\circ}{2}_{2}$ is an $\left(\varepsilon_{1}^{\prime}, 1\right)$-equivalence in $\left(N_{1}-\stackrel{\circ}{2}_{2}\right) \cup$ $(M-D)$. We can do this since our choice of $N_{1}$ can be made as close to $\infty$ as we want. It is easy to see that $\partial N_{1} \hookrightarrow N_{1}-\stackrel{\circ}{2}_{2}$ is an $\bar{\varepsilon}_{1}$-equivalence, and by the same argument it also follows that $\partial N_{2} \hookrightarrow N_{1}-\stackrel{\circ}{2}_{2}$ is an $\bar{\varepsilon}_{1}$-equivalence, where the size of $\bar{\varepsilon}_{1}$ depends on the size of $\varepsilon_{1}^{\prime}+\varepsilon_{1}^{\prime \prime}$. So all we have to do is choose $\varepsilon_{1}^{\prime}, \varepsilon_{1}^{\prime \prime}$ so that $\bar{\varepsilon}_{1} \leqslant \varepsilon_{1}$, and we are now ready for the next step of the construction.

II. $(m-3)$-Neighborhoods of $\infty$. In this step we want to show that the $N_{i}$ of step I can be chosen to additionally satisfy $\partial N_{i} \hookrightarrow N_{i}$ is $\left(m-3, \varepsilon_{i}\right)$-connected, i.e., $\left(N_{i}, \partial N_{i}\right)$ is $\left(m-3, \varepsilon_{i}\right)$-connected. The usual procedure is to inductively work up to the integer $m-3$ by showing that the $N_{i}$ can be constructed so that $\left(N_{i}, \partial N_{i}\right)$ is ( $\left.k, \varepsilon_{i}\right)$-connected, for $2 \leqslant k \leqslant m-3$. The case $k=2$ is the easiest to deal with. Given $\left\{N_{i}\right\}$ as in step I it will suffice to show how to rechoose $N_{1}$ to obtain $N_{1}^{\prime}$ for which $\partial N_{1}^{\prime} \hookrightarrow N_{1}^{\prime}$ is $\left(\varepsilon_{1}^{\prime}, 2\right)$-connected, for $\varepsilon_{1}^{\prime}$ a number whose size depends on the sizes of the $\varepsilon_{i}$. By Proposition 5.1 we can attach a finite number of 2-cells to $\partial N_{1}$ to form $K \subset N_{1}-N_{2}$ so that $\partial N_{1} \subset K$ and $K \hookrightarrow N_{1}$ is $(\varepsilon, 2)$-connected, where $\varepsilon$ depends on $\varepsilon_{1}$. Since $\partial N_{1} \hookrightarrow N_{1}-\stackrel{\circ}{2}_{2}$ is an $\left(\varepsilon_{1}, 1\right)$-equivalence we may choose the cells of $K-\partial N_{1}$ to be trivially attached, and we may choose these cells so that their images in $B$ are small. Let $R$ be a regular neighborhood of $\overline{M-N_{1}} \cup K$ and let $N_{1}^{\prime}=M-\stackrel{R}{ }$. Then $N_{1}^{\prime} \supset \supset N_{2} \supset \supset \cdots$ is a new sequence of neighborhoods of $\infty$. To see that $\partial N_{1}^{\prime} \hookrightarrow N_{1}^{\prime}$ $-\stackrel{\circ}{2}_{2}$ is an $\left(\varepsilon_{1}^{\prime}, 1\right)$-equivalence we use the fact that $K \hookrightarrow N_{1}$ is an $(\varepsilon, 1)$-equivalence along with general positioning to conclude that $\partial N_{1}^{\prime} \hookrightarrow N_{1}^{\prime}$ is an $\left(\varepsilon_{1}^{\prime \prime}, 1\right)$-equivalence, and this easily implies that $\partial N_{1}^{\prime} \hookrightarrow N_{1}^{\prime}-\stackrel{\circ}{2}_{2}$ is an $\left(\varepsilon_{1}^{\prime}, 1\right)$-equivalence. Since $\partial N_{2} \hookrightarrow N_{1}$ $-\stackrel{\circ}{N}_{2}$ is an $\left(\varepsilon_{1}, 1\right)$-equivalence we can similarly conclude that $\partial N_{2} \hookrightarrow N_{1}^{\prime}-\stackrel{\circ}{N}_{2}$ is an $\left(\varepsilon_{1}^{\prime}, 1\right)$-equivalence. Finally we again can use general positioning to prove that $\left(N_{1}^{\prime}, \partial N_{1}^{\prime}\right)$ is $\left(\varepsilon_{1}^{\prime}, 2\right)$-connected simply because $m \geqslant 6$. (Note that this is the last time that we will be able to use general positioning to show that $\left(N_{1}^{\prime}, \partial N_{1}^{\prime}\right)$ is $\left(\varepsilon_{1}^{\prime}, k\right)$ connected because the dimension restriction $m \geqslant 6$ is now critical.) This completes the construction of $N_{1}^{\prime}$ and therefore the proof of the case $k=2$.

The cases $3 \leqslant k \leqslant m-3$ are treated somewhat like $k=2$ above except that a new problem arises. Given $N_{1}$ for which $\left(N_{1}, \partial N_{1}\right)$ is $\left(\varepsilon_{1}, k-1\right)$-connected we can use Proposition 5.1 to form $K$ by attaching a finite number of $k$-cells to $\partial N_{1}$, and we can extend $\partial N_{1} \hookrightarrow N_{1}$ to an $(\varepsilon, k)$-connected map $f: K \rightarrow N_{1}$, where the size of $\varepsilon$ depends on the size of $\varepsilon_{1}$. The new problem we encounter is that in general we cannot embed $K$ in $N_{1}$ because of dimension restrictions. However the Whitney trick, along with the $\left(\varepsilon_{1}, k-1\right)$-connectivity of $\left(N_{1}, \partial N_{1}\right)$, does assure us that $K$ can be embedded as desired. Of course the heart of the Whitney trick is the existence of 2-discs which are then used to construct certain isotopies. In order for these isotopies to be small in $B$ these 2-discs must have small diameters in $B$. To achieve this one works locally, i.e., over open subsets of $B$ of small diameters, thus forcing these 2-discs to have small diameters in $B$. This is all explained in [7, §7.3]. Thus $K$ can be embedded in $N_{1}-N_{2}$ so that $K \hookrightarrow N_{1}$ is $(\varepsilon, k)$-connected. Now the proof proceeds as

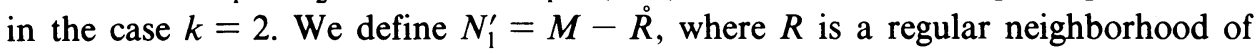


$\overline{M-N_{1}} \cup K$. Then $N_{1}^{\prime} \supset \supset N_{2} \supset \supset \cdots$ is a new sequence of neighborhoods of $\infty$ such that $\partial N_{1}^{\prime} \hookrightarrow N_{1}^{\prime}-\stackrel{\circ}{2}_{2}$ and $\partial N_{2} \hookrightarrow N_{1}^{\prime}-\stackrel{\circ}{2}_{2}$ are $\left(\varepsilon_{1}^{\prime}, 1\right)$-equivalences (by general positioning). To see that $\left(N_{1}^{\prime}, \partial N_{1}^{\prime}\right)$ is $\left(\varepsilon_{1}^{\prime}, k\right)$-connected we use the fact that $\left(N_{1}, K\right)$ is $(\varepsilon, k)$-connected along with excision and our Controlled Hurewicz Theorem, Theorem 3.1 (cf. [7, p. 318]).

III. $(m-2)$-Neighborhoods of $\infty$. It follows from step II above that for any $\varepsilon_{1}>\varepsilon_{2}>\cdots>0$ there are neighborhoods $N_{1} \supset \supset N_{2} \supset \supset \cdots$ of $\infty, \cap N_{i}=\varnothing$, so that $\left(N_{i}, \partial N_{i}\right)$ is $\left(\varepsilon_{i}, m-3\right)$-connected and $\partial N_{i+1} \hookrightarrow N_{i}-\stackrel{\circ}{N}_{i+1}$ is an $\left(\varepsilon_{i}, 1\right)$-equivalence. For any $\varepsilon>0$ we will show that there exists an $i$ and a neighborhood $N \subset N_{i}$ of $\infty$ for which $(N, \partial N)$ is $(\varepsilon, m-2)$-connected and $N \hookrightarrow N_{i}$ is an $(\varepsilon, 1)$-equivalence. Once we have established this it will follow that the sequence $\left\{N_{i}\right\}$ can be rechosen so that, additionally, $\left(N_{i}, \partial N_{i}\right)$ is $\left(\varepsilon_{i}, m-2\right)$-connected.

We begin by describing the problem that we are facing. Let us assume that the $N_{i}$ of step II exist, where $\lim \varepsilon_{i}=0$. Choose $i$ large and use Proposition 5.1 to extend $\partial N_{i} \hookrightarrow N_{i}$ to an $(\varepsilon, m-2)$-connected map $f: K \rightarrow N_{i}$, where $\varepsilon$ is small and $\left(K, \partial N_{i}\right)$ is a relative $\mathrm{CW}$ complex formed by attaching a finite number of $(m-2)$-cells to $\partial N_{i}$. Of course the problem is now to represent the images of these $(m-2)$-cells in $N_{i}$ by embedded $(m-2)$-cells, for once this is done they can be thickended to handles whose interiors can then be deleted from $N_{i}$ to yield our desired $N$ (cf. step II above). This representation problem is solved by relating $K$ to a handlebody structure on $N_{i}$, and then making use of Theorem 5.3 to alter this handlebody structure so that the $(m-2)$-cells are represented by a subset of the handles.

By the tameness at $\infty$ we can choose $j>i$ large enough so that there is a homotopy of $N_{i}$ into $N_{i}-\stackrel{\circ}{j}_{j}$ rel $\partial N_{i}$, where the image of this homotopy in $B$ is small. As in [7, §7.4] we may assume that the cobordism $\left(N_{i}-\stackrel{\circ}{N}_{j}, \partial N_{i}\right)$ consists only of $(m-3)$ - and $(m-2)$-handles with small diameters in $B$. Let $W$ be the union of $\partial N_{i}$ and the $(m-3)$-handles, and let $V=N_{i}-\stackrel{\circ}{N}_{j}$. From a homotopy point of view we may regard $\left(V, \partial N_{i}\right)$ as a relative $\mathrm{CW}$ complex which is formed by attaching $(m-3)$ - and $(m-2)$-cells to $\partial N_{i}$. Using the $\left(\varepsilon_{i}, m-3\right)$-connectivity of $\left(N_{i}, \partial N_{i}\right)$ we can find a map $r: N_{i} \rightarrow N_{i}$ for which $r(W)=\partial N_{i}, r \mid \partial N_{i}=$ id, and $r \simeq$ id rel $\partial N_{i}$ via a $p^{-1}\left(\varepsilon_{i}\right)$-homotopy. Proceeding as in the proof of Proposition 5.1 we can define our CW complex $K$ to be $V / \sim$, where $\sim$ is the equivalence relation generated by $x \sim r(x)$ for $x \in W$. Let $q: V \rightarrow K$ be the resulting quotient map. The map $f: K \rightarrow N_{i}$ is then defined by using levels of the homotopy $r \simeq$ id. Also let $s: N_{i} \rightarrow V$ be a map for which $s \mid \partial N_{i}=$ id and $s \simeq$ id rel $\partial N_{i}$ via a homotopy whose image in $B$ is small. Then $g=q s: N_{i} \rightarrow K$ is a map, and it is easy to see that $f g \simeq$ id rel $\partial N_{i}$ (with small control in $B$ ), i.e., $f$ is an $\varepsilon$-domination with inverse $g$. Of course, $f$ is also an $(\varepsilon, m-2)$-connected map.

The next step is to use Theorem 5.3. Since $p$ is nice at $\infty$ it is possible to find a compact relative $\mathrm{CW}$ complex $\left(P, \partial N_{i}\right)$ and an $\varepsilon$-equivalence $P \simeq N_{i}$ rel $\partial N_{i}$. This can be done for every $\varepsilon>0$ without the size of $\operatorname{dim} P$ increasing. (See Remark 2 following Theorem 2.3 of [2], and see the proof of the Controlled Boundary Theorem of [3].) Assume that the $(m-2)$-cells of $V$ are based at $\left(x_{i}\right)$ in $\partial N_{i}$, and so the $(m-2)$-cells of $K$ are also based at $\left(x_{i}\right)$. Let $A:\left(x_{i}\right) \rightarrow\left(x_{i}\right)$ be the $\varepsilon$-path matrix resulting from the map $g f: K \rightarrow K$. The conclusion of Theorem 5.3 requires that $A$ 
and $I-A$ be stabilized by identity matrices. $I-A$ can be stabilized by adding cancelling pairs of $(m-3)$ - and $(m-2)$-handles to $\partial N_{i} . A$ can be stabilized by adding cancelling pairs of $(m-3)$ - and $(m-2)$-handles to $\partial N_{i}$, and then deleting from $N_{i}$ the interiors of the ( $\left.m-3\right)$-handles. (This is exactly the situation encountered in [7, §7.4].) Assuming that such stabilizations have been carried out, Theorem 5.3 gives us an $\varepsilon$-path isomorphism $B:\left(x_{i}\right) \rightarrow\left(x_{i}\right)$ so that $A B$ and $(I-A) B$ are geometric. By this we mean that we can write $\left(x_{i}\right)=\left(\left(y_{j}\right),\left(z_{k}\right)\right)$ so that the columns of $A B$ corresponding to the elements $z_{k}$ are all 0 , and the columns of $(I-A) B$ corresponding to the elements $y_{j}$ are all 0 . We now interpret this data geometrically in $(V, W)$.

It is easy to see that $f q:(V, W) \rightarrow\left(N_{i}, \partial N_{i}\right)$ is homotopic to $r \mid:(V, W) \rightarrow\left(N_{i}, \partial N_{i}\right)$. Thus our problem of representing the images of the $(m-2)$-cells of $K$ in $N_{i}$ by embedded cells is equivalent to that of representing the images of the $(m-2)$-cells of $V$ in $N_{i}$ (under the map $r$ ) by embedded cells. Since $q s=g$ we have $q s r \simeq q s f q=$ $g f q$. But the path matrix of $q$ is the identity, so the path matrix of $s r:(V, W) \rightarrow(V, W)$ is just $A$. The next step is to dispose of $B$. According to the matrix identities on pp. 35-36 of [8] we can use $B$ to rechoose the handlebody structure of $V$ so that the columns of $A$ corresponding to the $z_{k}$ are 0 , and the columns of $I-A$ corresponding to the $y_{j}$ are 0 . This can be interpreted homotopically as follows. Let

$$
\phi_{j}:\left(I^{m-2}, \partial I^{m-2}, *\right) \rightarrow\left(V, W, y_{j}\right)
$$

be a characteristic map for the $(m-2)$-cells of $V$ based at $y_{j}$ and similarly let $\psi_{k}$ be a characteristic map for the $(m-2)$-cells based at $z_{k}$. Then we have homotopies $s r \phi_{j} \simeq \phi_{j}$ and $s r \psi_{k} \simeq *$ (constant map), where these homotopies are small in $B$. The first homotopy implies that each map $\phi_{j} \mid:\left(\partial I^{m-2}, *\right) \rightarrow\left(W, y_{j}\right)$ is homotopic to the constant map. The second homotopy implies that only the maps $r \phi_{j}$ have to be represented by embeddings into $N_{i}$.

Now using the argument of $[7, \S 7.4]$ each $\phi_{j} \mid: \partial I^{m-2} \rightarrow W$ (which is an embedding) is the 0 -level of an embedding of $\partial I^{m-2} \times[0,1]$ into $W$ so that the 1-level goes into $\partial N_{i}$. Thus the map $\phi_{j}$ extends to a characteristic map $\tilde{\phi}_{j}$ for an $(m-2)$-cell which is attached to $\partial N_{i}$. The $\tilde{\phi}_{j}$ are embeddings and they have disjoint images for different $j$. It is easy to check that $r \phi_{j} \simeq \tilde{\phi}_{j}$, for each $j$, and so our representation problem is solved. Thus $N$ can be constructed as desired.

IV. Conclusion of Proof. It follows from step III above that for any $\varepsilon_{1}>\varepsilon_{2}>$ $\cdots>0$ there are clean neighborhood $N_{1} \supset \supset N_{2} \supset \supset \cdots$ of $\infty, \cap N_{i}=\varnothing$, so that $\partial N_{i} \hookrightarrow N_{i}$ is $\left(\varepsilon_{i}, m-2\right)$-connected and $\partial N_{i+1} \hookrightarrow N_{i}-\stackrel{\circ}{i+1}_{i s}$ is $\left(\varepsilon_{i}, 1\right)$-equivalence. Since each $N_{i}-N_{i+1}^{\circ}$ can be given a handlebody structure with handles of only indices $m-3$ and $m-2$ whose images in $B$ are small, it easily follows from the tameness at $\infty$ that $\partial N_{i} \hookrightarrow N_{i}$ is an $\varepsilon_{i}^{\prime}$-equivalence, where $\lim \varepsilon_{i}^{\prime}=0$. By a local duality argument (as in [7, §5]) we conclude from Theorem 3.1 that $\partial N_{j} \hookrightarrow N_{i}-N_{j}$ is an $\varepsilon_{i}^{\prime \prime}$-equivalence, for any $i$, where $\lim \varepsilon_{i}^{\prime \prime}=0$. (This of course requires that the $N_{i}$ be chosen so that $\partial N_{j} \hookrightarrow N_{i}-\stackrel{\circ}{j}_{j}$ is an $\left(\varepsilon_{i}, 1\right)$-equivalence, for every $j>i$.) So summarizing we might as well assume that $\partial N_{i} \hookrightarrow N_{i}$ and $\partial N_{j} \hookrightarrow N_{i}-\stackrel{j}{j}_{j}$ are $\varepsilon_{i}$-equivalences, where $\lim \varepsilon_{i}=0$. We will use this information to show that $M$ admits a controlled boundary. 
Since $p$ is nice at $\infty$ it follows that for every $\varepsilon>0$ there exists a $\delta>0$ and an $i_{0}$ so that $p: N_{i} \rightarrow B$ is $(\varepsilon, \delta)$-nice, for all $i \geqslant i_{0}$. Thus for any given decreasing set $\left\{\delta_{j}\right\}_{j=1}^{k}$ of positive numbers we can find an $i_{1} \geqslant i_{0}$ large enough so that $p: N_{i} \rightarrow B$ is $\left(\delta_{j}, \delta_{j+1}\right)$-nice, for all $j$ and all $i \geqslant i_{1}$. Observe that by a sufficiently large choice of $i_{1}$ we can choose these $\delta_{j}$ 's small enough so that the Controlled $h$-Cobordism applies (see Addendum 2). So using the Controlled $h$-Cobordism Theorem it follows that for every $\varepsilon>0$ there exists an $i$ such that each cobordism $\left(N_{i}-\dot{\circ}_{j}, \partial N_{i}\right)$ is an $\varepsilon$-product, for all $j \geqslant i+1$. Now select $i_{1}<i_{2} \cdots$ so that $\left(N_{i_{n}}-{\stackrel{\circ}{i_{n+1}}}_{i_{n}}, \partial N_{i_{n}}\right)$ is an $\varepsilon_{n}$-product, where the sum $\sum \varepsilon_{n}$ is finite. Then piece together these $\varepsilon_{n}$-product structures to obtain a homeomorphism $h: \partial N_{i_{1}} \times[0,1) \rightarrow N_{i_{1}}$ such that $h(x, 0)=x$. By the finite condition it follows that $p h$ extends continuously to a map of $\partial N_{1} \times[0,1]$ to $B$. Thus $M$ admits a controlled boundary.

7. Proof of the controlled $h$-cobordism theorem. In [9] a proof is given of the proper $s$-cobordism theorem which uses a splitting technique to carve up a proper $h$-cobordism into a countable union of compact $h$-cobordisms. In our proof of the Controlled $h$-Cobordism Theorem we will use this splitting technique to carve up a controlled $h$-cobordism into a finite union of small $h$-cobordisms so that the usual uncontrolled $s$-cobordism theorem can then be applied to each of these small cobordisms. It will be helpful to recall the splitting technique that was used in [9], but first some notation. If $(X, Y)$ is a compact polyhedral $\underset{\varepsilon}{\text { pair }}$ and $p: X \rightarrow B$ is a controlling map to our parameter space, then the symbol $X \wedge Y$ means that there exists a compact polyhedron $K$ containing $X$ and $Y$ as subpolyhedra and PL retractions $\alpha: K \rightarrow X, \beta: K \rightarrow Y$ with contractible point-inverses such that $\alpha \simeq$ $\beta$ rel $Y$ via a $p^{-1}(\varepsilon)$-homotopy.

One-Dimensional Splitting Lemma. For every $\varepsilon>0$ and integer $m$ there exists $a$ $\delta>0$ so that if $\left(W^{m+1}, \partial_{0} W\right) \stackrel{p}{\rightarrow}[0,3]$ is a compact $(\delta, h)$-cobordism and $W \stackrel{\delta}{\wedge} \partial_{0} W$, then there exists a clean submanifold $V$ of $W$ for which

(1) $p^{-1}([0,1]) \subset V \subset p^{-1}([0,2])$,

(2) $\left(V, V \cap \partial_{0} W\right) \rightarrow[0,3]$ is an $(\varepsilon, h)$-cobordism,

(3) $\left(\overline{W-V}, \overline{W-V} \cap \partial_{1} W\right) \rightarrow[0,3]$ is an $(\varepsilon, h)$-cobordism.

(In this statement we use $\partial_{1} W$ for $\overline{\partial W-\partial_{0} W}$, and it should be noted that the parameter space is $[0,3]$.)

Very briefly the above result is established by using the methods of the proof of the boundary theorem of [8] to obtain the splitting. As explained in [9, p. 492] we can carry this out provided that given any clean submanifold $V$ of $W$ for which

$$
p^{-1}([0,1.5-\gamma]) \subset V \subset p^{-1}([0,1.5])
$$

and $\gamma$ is small, then the inclusion $\overline{\partial V-\partial_{0} W} \hookrightarrow V$ can be extended to a homotopy equivalence by the addition of a compact polyhedron to

$$
\overline{\partial V-\partial_{0} W} \cap p^{-1}([1.5-2 \gamma, 1.5]) \text {. }
$$


$\delta$

But for $\delta$ sufficiently small the condition $W \wedge \partial_{0} W$ guarantees that this can be done.

$\delta$

More generally if the property $W \wedge \partial_{0} W$ is not satisfied, then the obstruction to finding such an extension is a relative Wall finiteness obstruction as appears in the first exact sequence of [9] for analyzing infinite simple types.

In order to adapt the above ideas to our situation in which the parameter space is more complicated we will have to sharpen the above definition. For $(X, Y)$ and $p: X \rightarrow B$ as above, the symbol. $X \wedge Y$ means that there exists $K, \alpha$ and $\beta$ as above for which $\operatorname{dim} K \leqslant n$. Now for any $B$ let $\phi: B \rightarrow[0,3]$ be a map. Here is our general splitting result.

General Splitting Lemma. For any $B$ let $\varepsilon>0$ and $n$ be given. There exists a $\delta>0$ so that if $\left(W, \partial_{0} W\right) \stackrel{p}{\rightarrow} B$ is a compact $(\delta, h)$-cobordism and $W \bigwedge_{n}^{\delta} \partial_{0} W$, then there exists a clean submanifold $V$ of $W$ for which

(1) $(\phi p)^{-1}([0,1]) \subset V \subset(\phi p)^{-1}([0,2])$,

(2) $\left(V, V \cap \partial_{0} W\right) \stackrel{p}{\rightarrow} B$ is an $(\varepsilon, h)$-cobordism,

(3) $\left(\overline{W-V}, \overline{W-V} \cap \partial_{1} W\right) \stackrel{p}{\rightarrow} B$ is an $(\varepsilon, h)$-cobordism.

In analogy with the One-Dimensional Splitting Lemma this result is established by using the methods of proof of our Controlled Boundary Theorem. Note that we are not guilty of circular reasoning, for we use only the first part of the proof which did not make use of the Controlled $h$-Cobordism Theorem. It should be clear why the controlling integer $n$ is needed, for it was also needed in the proof of the Controlled Boundary Theorem to guarantee the small $\varepsilon$-control in $B$.

There is one more ingredient we will need for the proof of the main result of this section. It is the Approximation Theorem from [2].

ApProximation TheOrem [2]. Given integers $m$ and $b$ there exists an integer $n$ which satisfies the following property: for every $B$ and $\gamma>0$ there exists a decreasing set $\left\{\gamma_{i}\right\}_{i=1}^{l}$ of positive numbers so that if $(X, Y)$ is a compact polyhedral pair, $p: X \rightarrow B$ is $\left(\gamma_{i}, \gamma_{i+1}\right)$-nice for each $i, \operatorname{dim} X \leqslant m$ and $\operatorname{dim} B \leqslant b$, and $Y \hookrightarrow X$ is a $\gamma_{1}$-equivalence, then $X \underset{n}{\wedge} Y$.

REMARKS. 1. It is important to note that $l$ depends only on $\operatorname{dim} B$, and the $\gamma_{i}$ can be rechosen in the same manner that the $\delta_{i}$ of the Controlled $h$-Cobordism can be rechosen (see Addendum 2 of $\$ 1$ ).

2. There is also a relative version of the above result, where the assumption that $p$ be $\left(\gamma_{i}, \gamma_{i+1}\right)$-nice is replaced by the somewhat weaker condition that there exists a decreasing sequence $\left\{X_{i}\right\}_{i=1}^{l}$ of closed subpolyhedra of $X$ for which

(1) $X_{l} \cap Y \hookrightarrow X_{l}$ is a $\gamma_{\Gamma}$ equivalence,

(2) $X-X_{l} \subset Y$, 
(3) for each $b \in B$ and $i$, the inclusion

$$
\left(p^{-1}\left(S_{\gamma_{i+1}}(b)\right) \cap X_{i+1}\right) \times T^{k} \hookrightarrow\left(p^{-1}\left(S_{\gamma_{i}}(b)\right) \cap X_{i}\right) \times T^{k}
$$

induces the 0 -homomorphism on Whitehead groups, for all $k$. (We say that $p$ is $\left(\gamma_{i}, \gamma_{i+1}\right)$-nice on $\left(X_{i}, X_{i+1}\right)$.)

We are now ready to begin the proof of the Controlled $h$-Cobordism Theorem. It will be convenient to first assume that $B$ is a compact polyhedron, $\operatorname{dim} B=b$. For our given $m+1$ we have the integers $n$ and $l$ of the Approximation Theorem. For our choice of $k$ we will use $k=l b$. We will show that if the $\left\{\delta_{i}\right\}_{i=1}^{k}$ are chosen appropriately and $p: W^{m+1} \rightarrow B$ is $\left(\delta_{i}, \delta_{i+1}\right)$-nice, then $\left(W, \partial_{0} W\right)$ is an $\varepsilon$-product. For notation choose a subdivision of $B$ which is fine enough so that all the simplices have small diameters with respect to $\varepsilon$. $B^{(r)}$ denotes the $r$ th barycentric subdivision of $B$ and $B^{i}$ denotes the $i$ skeleton of $B$. Also $\operatorname{St}\left(B^{i}, B^{(r)}\right)$ is the star of $B^{i}$ in the $r$ th barycentric subdivision, which we abbreviate as $B_{r}^{i}$.

Now for the first step we can use the Approximation Theorem and the General Splitting Lemma to find a clean submanifold $W_{b-1}$ of $W$ for which

(1) $B_{5}^{b-1} \subset p\left(W_{b-1}\right) \subset B_{2}^{b-1}$,

(2) $\left(W_{b-1}, W_{b-1} \cap \partial_{0} W\right) \rightarrow B$ and $\left(\overline{W-W}_{b-1}, \overline{W-W}_{b-1} \cap \partial_{1} W\right) \rightarrow B$ are $\left(\delta_{l(b-1)}, h\right)$-cobordisms.

This requires that the number $\delta_{l(b-1)}$ must correspond to the number $\gamma$ of the Approximation Theorem, and the set $\left\{\delta_{i}\right\}_{i=l(b-1)+1}^{k}$ must correspond to the set $\left\{\gamma_{i}\right\}_{i=1}^{l}$. For convenience we may assume that $p$ is PL and each $p^{-1}\left(B_{r}^{b-1}\right)$ is clean. Thus the above splitting can be trivially extended to additionally satisfy the property that $W_{b-1} \cap p^{-1}\left(\overline{B_{2}^{b-1}-B_{3}^{b-1}}\right)$ is a small collar on $p^{-1}\left(\overline{B_{2}^{b-1}-B_{3}^{b-1}}\right) \cap$ $\partial_{0} W$ and $\overline{W-W}_{b-1} \cap p^{-1}\left(\overline{B_{4}^{b-1}-B_{5}^{b-1}}\right)$ is a small collar on $p^{-1}\left(\overline{B_{4}^{b-1}-B_{5}^{b-1}}\right) \cap$ $\partial_{1} W$. If $\left\{\Delta_{i}^{b}\right\}$ is the collection of $b$-cells in $B$, then the $h$-cobordism $\left(\overline{W-W}_{b-1}\right.$, $\left.\overline{W-W}_{b-1} \cap \partial_{1} W\right)$ is a disjoint union of small $h$-cobordisms $\left(V_{i}, V_{i} \cap \partial_{1} W\right)$, where $V_{i}=p^{-1}\left(\Delta_{i}^{b}\right) \cap \overline{W-W}_{b-1}$. By the relative version of the Approximation Theorem we conclude that the Whitehead torsion of the $h$-cobordism $\left(V_{i}, V_{i} \cap \partial_{1} W\right)$ is 0 (this is the reason why the above splitting was extended). Now extend $\left(W_{b-1}, W_{b-1} \cap \partial_{0} W\right)$ to a $\left(\delta_{l(b-1)}, h\right)$-cobordism $\left(\tilde{W}_{b-1}, \partial_{0} W\right)$ by adding to $W_{b-1}$ a small collar on $\partial_{0} W$. The triviality of the $h$-cobordism $\left(V_{i}, V_{i} \cap \partial_{1} W\right)$ gives us a homeomorphism $h_{1}$ of $W$ onto $\tilde{W}_{b-1}$ which is supported on $p^{-1}\left(\overline{B-B_{5}^{b-1}}\right)$.

This completes the first step of our construction which succeeds in replacing $\left(W, \partial_{0} W\right)$ by $\left(\tilde{W}_{b-1}, \partial_{0} W\right)$, where $\tilde{W}_{b-1}$ contains the portion of $W$ that lies over some small regular neighborhood of $B^{b-1}$, and $\tilde{W}_{b-1}$ is a small collar on $\partial_{0} W$ over the complement of some larger regular neighborhood of $B^{b-1}$. The next step is to replace $\left(\tilde{W}_{b-1}, \partial_{0} W\right)$ by $\left(\tilde{W}_{b-2}, \partial_{0} W\right)$, where $\tilde{W}_{b-2}$ contains the portion of $W$ that lies over some small regular neighborhood of $B^{b-2}$ and $\tilde{W}_{b-2}$ is a small collar on $\partial_{0} W$ over the complement of some larger regular neighborhood of $B^{b-2}$. This is accomplished by applying the above splitting ideas to the cobordism $\left(\tilde{W}_{b-1}, \partial_{0} W\right)$. We then obtain a homeomorphism $h_{2}: \tilde{W}_{b-1} \rightarrow \tilde{W}_{b-2}$ which is the identity over a regular neighborhood of $B^{b-2}$ and over the complement of a regular neighborhood of $B^{b-1}$. More specifically, the support of $h_{2}$ lies over a small regular neighborhood 
of the set of barycenters of the $(b-1)$-simplices of $B$. This completes the second step of the construction.

Now continuing the above process we can construct controlled $h$-cobordisms $\left(\tilde{W}_{i}, \partial_{0} W\right)$ and homeomorphisms

$$
W \stackrel{h_{1}}{\rightarrow} \tilde{W}_{b-1} \stackrel{h_{2}}{\rightarrow} \tilde{W}_{b-2} \rightarrow \cdots \stackrel{h_{b}}{\rightarrow} \tilde{W}_{0},
$$

where $\left(\tilde{W}_{0}, \partial_{0} W\right)$ is a small collar on $\partial_{0} W$ over the complement of some regular neighborhood of $B^{0}$. Thus $\left(\tilde{W}_{0}, \partial_{0} W\right)$ is essentially a union of small $h$-cobordismsone for each vertex of $B$. By the relative version of the Approximation Theorem each one of these $h$-cobordisms is trivial. Thus we have a homeomorphism $k: \tilde{W}_{0} \rightarrow \partial_{0} W$ $\times[0,1]$ and $h=k h_{b} \cdots h_{1}: W \rightarrow \partial_{0} W \times[0,1]$ is a homeomorphism as desired. It is clear that if the regular neighborhoods are appropriately chosen, then we must have $\varepsilon$-control on $h$ because for each point $x$ of $W$ we must have $p(x)$ and $p h(x)$ in the star of some simplex. This completes the proof of the polyhedral case.

The proof of the general case is just an easy reduction to the polyhedral case. For $B$ a finite-dimensional compact metric space choose $B \subset B^{\prime}$, where $B^{\prime}$ is a compact polyhedron, and let $p^{\prime}: W \rightarrow B^{\prime}$ be the composition $W \stackrel{p}{\rightarrow} B \hookrightarrow B^{\prime}$. Then one easily shows that the above case, which deals with the controlled $h$-cobordism $\left(W, \partial_{0} W\right) \stackrel{p^{\prime}}{\rightarrow} B^{\prime}$, implies that $\left(W, \partial_{0} W\right) \stackrel{p}{\rightarrow} B$ is an $\varepsilon$-product. It should be mentioned that if $k^{\prime}$ is the integer which described how many of the $\delta_{i}$ are needed for $B^{\prime}$, then $k=3 k^{\prime}-2$ describes how many of the $\delta_{i}$ are needed for $B$ (cf. the proof of Lemma 7.1 of $[2])$.

\section{REFERENCES}

1. H. Bass, A. Heller and R. Swan, The Whitehead group of a polynomial extension, Inst. Hautes Études Sci. Publ. Math. 22 (1964), 67-79.

2. T. A. Chapman, A general approximation theorem for Hilbert cube manifolds, preprint.

3. A controlled boundary theorem for Hilbert cube manifolds, preprint.

4. T. A. Chapman and Steve Ferry, Approximating homotopy equivalences by homeomorphisms, Amer. J. Math. 101 (1979), 583-607.

5. M. Cohen, A course in simple-homotopy theory, Springer-Verlag, New York, 1970.

6. R. Kirby and L. Siebenmann, Foundational essays on topological manifolds, smoothings, and triangulations, Ann. of Math. Studies, no. 88, Princeton Univ. Press, Princeton, N. J., 1977.

7. Frank Quinn, Ends of maps. I, Ann. of Math. (2) 110 (1979), 275-331.

8. L. Siebenmann, The obstruction to finding a boundary for an open manifold of dimension greater than five, Thesis, Princeton Univ. Press, Princeton, N. J., 1965.

9. Infinite simple homotopy types, Indag. Math. 32 (1970), 479-495.

10. C. T. C. Wall, Finiteness conditions for CW complexes, Ann. of Math. (2) 81 (1965), 55-69.

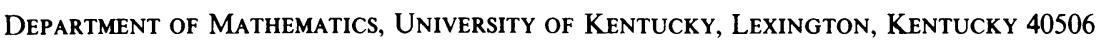

